\title{
Compliance im Gesellschaftsrecht
}

Gerald Spindler*

A. Einleitung ........................ 292

B. (Gesellschafts)rechtliche Grundlagen ............................... 293

C. Compliance im Finanz- und Kapitalmarktaufsichtsrecht .................. 296 I. Spezialgesetzliche Vorgaben (WpHG) . . 296

II. Kreditinstitute ..................... 299

III. Versicherungsunternehmen ......... 301

D. IDW-Prüfstandards .................. 302

E. Folgerungen für die AG ............. 305

F. Rechtsfolgen bei Verstoß, insbesondere Haftung. ........................... 309

I. Haftung nach $\$ 93$ Abs. 1 AktG, insbesondere Business Judgement

Rule....
II. Haftung gegenüber Dritten und strafrechtliche Verantwortlichkeit... 313

G. Konzernbezogene Compliance-Anforderungen ........................... 315

I. Konzernbezogene Organisationspflichten?........................ 315

II. Konzernbezogene Compliance....... 318

III. Datenschutzrechtliche Grenzen...... 321

H. Übertragung auf andere Gesellschaftsformen............................. 323

I. Nötige empirische Untersuchungen . . 323

J. Kritisches Fazit ..................... 324

\section{A. Einleitung}

Gäbe es eine „Hitliste“ der juristischen Themen im Gebiet der Beratung, das Zauberwort der „Compliance“ würde mit Sicherheit die Liste anführen. Kaum ein Schlagwort hat Seminare, Konferenzen und Beratungen derart beherrscht wie die „Compliance“; selbst führende Verlage sahen sich veranlasst, neue Zeitschriften zu gründen, um dem offenbar großen Beratungsbedürfnis gerecht zu werden. ${ }^{1}$ Scharen von Praktikern publizieren Aufsatz um Aufsatz, Handbuch um Handbuch ${ }^{2}$ und entdecken stets aufs Neue „Compliance“-Lücken, die es zu füllen gilt. Neue Stellen in Gestalt von „Compliance“-Officern wurden geschaffen nebst ganzen Abteilungen, die der Umsetzung der „Compliance“ im Unternehmen dienen soll. Quasi jeder Rechtsbereich wird jetzt um eine „Compliance“-Funktion ergänzt, ${ }^{3}$ sofern auch nur irgendein Verstoß eines Unternehmens gegen ein wie auch immer geartetes Gesetz drohen könnte.

* Prof. Dr. Gerald Spindler ist Inhaber des Lehrstuhls für Bürgerliches Recht, Handels- und Wirtschaftsrecht, Rechtsvergleichung, Multimedia- und Telekommunikationsrecht an der Universität Göttingen.

1 S. etwa die „CCZ“ des Beck-Verlages alias „Corporate Compliance Zeitschrift“ oder „CB“ des deutschen Fachverlages alias „Compliance Berater“.

2 Statt vieler etwa C. Hauschka (Hrsg)., Corporate Compliance: Handbuch der Haftungsvermeidung im Unternehmen, 2. Aufl., München 2010.

3 Zur Kartellrechtscompliance M. Dreher, Kartellrechtscompliance - Voraussetzungen und Rechtsfolgen unternehmens- oder verbandsinterner Maßnahmen zur Einhaltung des Kartellrechts, ZWeR 2004, S. 75; ders. Kartellrechtscompliance in der Versicherungswirtschaft, VersR 2004, S. 1; zu den Compliance-Pflichten im WpHG s. unten C. I. sowie U.H. Schneider/J. v. Buttlar, Die Einführung von Insiderverzeichnissen: Neue Compliance-Pflichten für Emittenten, ZIP 2004, S. 1621; zur Prävention gegen strafrechtliche Haftung durch Compliance nach US-Recht W. Walisch, Organisatorische Prävention gegen strafrechtliche Haftung deutscher Unternehmen und ihrer Leitungen nach US-Recht, Berlin 2004, S. 55 . 
Der nachfolgende Beitrag unternimmt eine kritische Bestandsaufnahme, die allerdings angesichts der kaum zu überblickenden Flut an Beiträgen zur Compliance keineswegs Vollständigkeit für sich beansprucht. Vielmehr wird den Wurzeln der Compliance-Bewegung nachgespürt, insbesondere im Gesellschafts- und Finanzaufsichtsrecht, die in den größeren Zusammenhang der Organisationspflichten zu stellen sind.

\section{B. (Gesellschafts)rechtliche Grundlagen}

Compliance stellt bei Lichte gesehen eine Selbstverständlichkeit dar - nämlich schlicht die Pflicht, bestehende Vorschriften einzuhalten. Diese jedermann treffende Pflicht gilt natürlich auch für alle arbeitsteiligen Organisationen, insbesondere auch juristische Personen, ohne dass dies bislang einer besonderen Erwähnung bedurft hätte. ${ }^{4}$ Compliance drückt dabei lediglich die auf Organisationen übertragene Pflicht aus, dafür zu sorgen, dass Rechtsverstöße durch die Organisation (bzw. Unternehmen oder juristische Person) unterbleiben. Dies mag man als Ausdruck der etwas euphemistisch bezeichneten Legalitätspflicht, ${ }^{5}$ als Element der in $\mathbb{9} 91 \mathrm{AktG}^{6}$ statuierten Pflicht zum Risikomanagement ${ }^{7}$ oder gar als Ausfluss der Anforderungen im

4 Näher dazu G. Spindler, Die Haftung von Vorstand und Aufsichtsrat für fehlerhafte Auslegung von Rechtsbegriffen, in: A. Heldrich/J. Prölls/I. Koller et al.(Hrsg), Festschrift für Claus-Wilhelm Canaris zum 70. Geburtstag, Band II, München 2007, S. 403 (412).

5 H.-J. Mertens/A. Cahn, in: W. Zöllner/U.Noack (Hrsg.), Kölner Kommentar zum Aktiengesetz, Band 2/1, 3. Aufl., Köln 2010, $\$ 91$ Rn. 35; G. Bachmann, Compliance - Rechtsgrundlagen und offene Fragen, in: Gesellschaftsrechtliche Vereinigung (Hrsg.), Gesellschaftsrecht in der Diskussion 2007 - Jahrestagung der gesellschaftsrechtlichen Vereinigung (VGR), Köln 2008, S. 65 (73); H. Fleischer, Kartellrechtsverstöße und Vorstandsrecht, BB 2008, S. 1070 (1071).; ders., Corporate Compliance im aktienrechtlichen Unternehmensverbund, CCZ 2008, S. 1; J. Reichert/ N. Ott, Non Compliance in der AG Vorstandspflichten im Zusammenhang mit der Vermeidung, Aufklärung und Sanktionierung von Rechtsverstößen, ZIP 2009, S. 2173 (2174); W. Goette, Organisationspflichten in Kapitalgesellschaften zwischen Rechtspflicht und Opportunität, ZHR 175 (2011), S. 388 (392); D. Verse, Compliance im Konzern - Zur Legalitätskontrollpflicht der Geschäftsleiter einer Konzernobergesellschaft, ZHR 175 (2011), S. 401 (403 f.); E. Bicker, Compliance - organisatorische Umsetzung im Konzern, AG 2012, S. 542 (543 f.); B. Reese/C. Ronge, Aufgaben und Struktur der Compliance-Funktion im Versicherungsunternehmen unter besonderer Berücksichtigung von Solvency II, VersR 2011, S. 1217 (1220); B. Schmidt, Vertrauen ist gut, Compliance ist besser! - Anforderungen an die Datenverarbeitung im Rahmen der Compliance-Überwachung, BB 2009, S. 1295 f.; D. Meier-Greve, Vorstandshaftung wegen mangelhafter Corporate Compliance, BB 2009, S. 2555; G. Kammerer-Galahn, Compliance - Herausforderung für Unternehmensleiter und deren Rechtsberater, AnwBl. 2009, S. 77; M. Sänger, Whistleblowing in der börsennotierten Aktiengesellschaft, Frankfurt 2010, S. 222; wohl auch U. Bergmoser/I. Theusinger/K. Gushurst, Corporate Compliance - Grundlagen und Umsetzung, BB Beilage 2008, S. 1 (5); kritisch zum Ganzen wegen zu restriktiver Auslegung U.H. Schneider, Compliance im Konzern, NZG 2009, S. 1321 (1323).

6 Zur Frage der Übertragbarkeit auf andere Rechtsformen s. unten H.

7 M. Dreher, Die Vorstandsverantwortung im Geflecht von Risikomanagement, Compliance und interner Revision in: P. Kindler/J. Koch/P. Ulmer/M. Winter (Hrsg.), Festschrift für Uwe Hüffer zum 70. Geburtstag, München 2010, S. 161 (168 ff.) mwN; ders, Ausstrahlung des Aufsichtsrechts auf das Aktienrecht, ZGR 2010, S. 496 (519); G. Spindler, Compliance in der multinationalen Bankengruppe, WM 2008, S. 905 (906 f.); C. Berg, Korruption in Unternehmen und Risikomanagement nach \91 Abs. 2 AktG, AG 2007, S. 271 (271); wohl auch W. Schaupensteiner, Rechtstreue im Unternehmen - Compliance und Krisenmanagement - Konzertiertes Vorgehen statt einzelbetriebliche Maßnahmen, NZA-Beilage 2011, S. 8 (10). 
Außenverhältnis ( $\$ 130 \mathrm{OWiG})$ dieser Organisationspflicht ansehen; ${ }^{8}$ der Inhalt, nämlich die arbeitsteilige Organisation so zu strukturieren, dass möglichst keine Rechtsverstöße von ihr ausgehen, bleibt der Gleiche. Dementsprechend besteht Einigkeit, dass der Vorstand das Unternehmen den gesetzlichen Bestimmungen gemäß zu organisieren und zu führen hat. ${ }^{9}$ Dies ergibt sich nicht zuletzt aus der Abschlussprüfer-Richtlinie, die ein Überwachungssystem fordert, das auch die Compliance umfassen soll. ${ }^{10}$ Der Vorstand ist nach ganz hM daher im Rahmen seiner aktienrechtlichen Überwachungssorgfalt nach $\$ 93$ Abs. 1 AktG dafür verantwortlich, gerade in besonders für Verstöße anfälligen Unternehmensbereichen geeignete Vorkehrungen zur Früherkennung von Risiken zu treffen, wozu eben auch rechtliche Risiken gehören. ${ }^{11}$

8 J. Koch, Compliancepflichten im Unternehmensverbund?, WM 2009 S. 1013 ff.; M. Rieder/M. Jerg, Anforderungen an die Überprüfung von Compliance-Programmen, CCZ 2010, S. 201; K. von Busekist/C. Schlitt, Der IDW PS 980 und die allgemeinen rechtlichen Mindestanforderungen an ein wirksames Compliance Management System (2) - Risikoermittlungspflicht, CCZ 2012, S. 86 f.; K. von Busekist/O. Hein, Der IDW PS 980 und die allgemeinen rechtlichen Mindestanforderungen an ein wirksames Compliance Management System (1) - Grundlagen, Kultur und Ziele, CCZ 2012, S. 41 (43).

9 H. Fleischer, in: G. Spindler/E. Stilz (Hrsg.), Kommentar zum Aktiengesetz, Band I, 2. Aufl., München 2010, $\$ 91$ Rn. 50; M. Kort, in: K. J. Hopt/ H. Wiedemann (Hrsg.), Großkommentar zum Aktiengesetz, Bd. 3, 4. Aufl., Berlin 2008, $\mathbb{S} 91$ Rn. 65; T. Lösler, Das moderne Verständnis von Compliance im Finanzmarktrecht, NZG 2005, S. 104 (105); C. Hauschka, Compliance, Compliance-Manager, Compliance-Programme: Eine geeignete Reaktion auf gestiegene Haftungsrisiken für Unternehmen und Management?, NJW 2004, S. 257; ders., Corporate Compliance - Unternehmensorganisatorische Ansätze zur Erfüllung der Pflichten von Vorständen und Geschäftsführern, AG 2004, S. 461 (462); ders., Compliance am Beispiel der Korruptionsbekämpfung - Eine Erwiderung aus der Praxis auf Uwe H. Schneiders Vorschläge, ZIP 2004, S. 877 (877 f.); H. Fleischer, Vorstandsverantwortlichkeit und Fehlverhalten von Unternehmensangehörigen - Von der Einzelüberwachung zur Errichtung einer Compliance-Organisation, AG 2003, S. 291 (299); J. Bürkle, Weitergabe von Informationen über Fehlverhalten im Unternehmen (Whistleblowing) und Steuerung auftretender Probleme durch ein Compliance-System, DB 2004, S. 2158 (2160); D. Eisele, Insiderrecht und Compliance, WM 1993, S. 1021 (1023); ausführlich zur Errichtung einer Compliance-Organisation G. Spindler, Vorstandspflichten zur Einrichtung eines Frühwarnsystems, in: H. Fleischer (Hrsg.), Handbuch des Vorstandsrechts, 1. Aufl., München 2006, $\$ 19$ Rn. 51 ff.; J. Bürkle, Corporate Compliance - Pflicht oder Kür für den Vorstand der AG?, BB 2005, S. 565 ff.; J. Rodewald/U. Unger, Corporate-Compliance - Organisatorische Vorkehrungen zur Vermeidung von Haftungsfällen der Geschäftsleitung, BB 2006, S. 113; zusammenfassend M. Winter, Die Verantwortlichkeit des Aufsichtsrats für „Corporate Compliance“, in: P. Kindler et al. (Hrsg.), FS Hüffer (Fn. 7), S. 1103 (1104 f.).

10 Hierzu auch G. Spindler, Von der Früherkennung von Risiken zum umfassenden Risikomanagement - zum Wandel des $\mathbb{9} 91$ AktG unter europäischem Einfluss, in: P. Kindler et al. (Hrsg.), FS Hüffer (Fn. 7), S. 985 (992).

11 Fleischer, Vorstandsverantwortlichkeit (Fn.9), S. 299; U.H. Schneider, Compliance als Aufgabe der Unternehmensleitung, ZIP 2003, S. 645 (649); im Überblick M. Kort, Verhaltensstandardisierung durch Corporate Compliance, NZG 2008, S. 81 ff.; Bergmoser/Theusinger/Gushurst, Corporate Compliance (Fn. 5), S. 1; Mertens/Cahn (Fn. 5), $\$ 91$ Rn. 34; alle mwNachw. 
Angesichts der äußerst hohen Geldbußen und Schadensersatzzahlungen verwundert es nicht, dass im Zentrum der Compliance-Überlegungen vor allem das Kartellrecht $^{12}$ steht, aber auch der Komplex der kapitalmarktrechtlichen Informationspflichten, die schließlich in einer persönlichen Haftung der Organe münden können. ${ }^{13}$ Daneben spielt das Anwendungsfeld der Bestechungszahlungen gerade im Bereich der Auftragsvergabe eine bedeutende Rolle. ${ }^{14}$ Ferner hat der Bereich der Datenschutz-Compliance inzwischen eine ebenso große Bedeutung erlangt. ${ }^{15}$ Alle Bereiche zeichnen sich durch eine hohe Öffentlichkeitssensibilität, persönliche Haftungs- und Strafbarkeitsrisiken sowie potentielle Schäden für die Unternehmen aus.

Während über das „Ob“ einer Compliance-Pflicht wenig Streit besteht, da eben jedermann, damit auch Organisationen zur Einhaltung von Recht und Gesetz verpflichtet sind, herrscht hinsichtlich der Frage des „Wie“ große Unsicherheit. Abgesehen davon, ob die Geschäftsleitung hier ein unternehmerisches Ermessen für sich in Anspruch nehmen kann (Business Judgement Rule, $\mathbb{\int} 93$ Abs. 1 S. 2 AktG), ${ }^{16}$ ist schon oftmals unklar, welche konkreten Anforderungen überhaupt bestehen. Gleichzeitig zeigen sich hier erhebliche Tendenzen zur Verrechtlichung der (Unterneh-

12 M. Zimmermann, Kartellrechtliche Bußgelder gegen Aktiengesellschaften und Vorstand: Rückgriffsmöglichkeiten, Schadensumfang und Verjährung, WM 2008, S. 433 (435); T. Lampert, Gestiegenes Unternehmensrisiko Kartellrecht - Risikoreduzierung durch Competition - Compliance-Programme, BB 2002, S. 2237 ff., U. Itzen, Richtungswechsel, Bestandsaufnahme, Prävention: Das Gerüst einer erfolgreichen Compliance-Strategie, BB 2008, Beil. 005, S. 12 (14 ff.); Fleischer, Kartellrechtsverstöße (Fn. 5), S. 1072; ders., Corporate Compliance (Fn. 5), S. 2; P. V. van Vormizeele, Kartellrechtliche Compliance-Programme im Rahmen

der Bußgeldbemessung de lege lata und de lege ferenda, CCZ 2009, S. 41 (42); G. Pampel, Die Bedeutung von Compliance-Programmen im Kartellordnungswidrigkeitenrecht, BB 2007, S. 1636 ff.; C. Hauschka, Der Compliance-Beauftragte im Kartellrecht, BB 2004, S. 1178 ff.; M. Dreher, Kartellrechtscompliance (Fn. 3), S. 76 ff.; J. Schmidt/A. Koyuncu, Kartellrechtliche Compliance-Anforderungen an den Informationsaustausch zwischen Wettbewerbern, BB 2009, S. 2551 (2554); Rieder/Jerg, Überprüfung (Fn. 8), S. 203; Kort, Verhaltensstandardisierung (Fn. 11), S. 83; T. Lampert/P. Matthey (Fn. 2), \$26 Rn. 48 ff.; W. Hölters, in: W. Hölters (Hrsg.), Aktiengesetz Kommentar, 1. Aufl., München 2011, $\$ 93$ Rn. 112.

13 So auch Schneider, Compliance (Fn. 11), S. 647 ff., H. Wendel, Kapitalmarkt Compliance in der Praxis - Anforderungen an die Organisation in börsennotierten Unternehmen zur Erfüllung insiderrechtlicher Pflichten, CCZ 2008, S. 41 (45 ff.); Fleischer, Vorstandsverantwortlichkeit (Fn. 9), S. 299; zum gestiegenen Haftungsrisiko J. Buchta, Die Haftung des Aufsichtsrats einer Aktiengesellschaft - aktuelle Entwicklungen in Gesetzgebung und Rechtsprechung Teil 1, DStR 2003, S. 694 (697 ff.) (740 ff.); T. Baums, Haftung wegen Falschinformation des Sekundärmarktes, ZHR 167 (2003), S. 139 ff.; P. BuckHeeb, Informationsorganisation im Kapitalmarktrecht Compliance zwischen Informationsmanagement und Wissensorganisationspflicht, CCZ 2009, S. $18 \mathrm{ff} . ;$ Wendel, Kapitalmarkt Compliance (Fn. 13), S. 41 ff.; D. Eisele/Faust, in: H. Schimansky/H.-J. Bunte/H.-J. Lwowski (Hrsg.), BankrechtsHandbuch, 4. Aufl., München 2011, $\mathbb{1 0 9}$ Rn. $42 \mathrm{ff}$.

14 C. Hauschka/G. Greeve, Compliance in der Korruptionsprävention - was müssen, was sollen, was können die Unternehmen tun?, BB 2007, S. 165; S. Salvenmoser/C. Hauschka, Korruption, Datenschutz und Compliance, NJW 2010, S. 331 (334); G. Greeve, (Fn. 2), $\$ 25$ Rn. 126 ff.; Hölters (Fn. 12), \93 Rn. 113.

15 Siehe z.B. Salvenmoser/Hauschka, Korruption (Fn. 14), S. 332 f.; F. Maschmann, Compliance versus Datenschutz, NZA-Beilage 2012, S. 50 ff.

16 Dazu unten F. I. 
mens-) Organisation, die eine Entwicklung der letzten Jahrzehnte fortschreiben ${ }^{17}$ und quasi zur „neuen Blüte“ treiben. Charakteristisch hierfür sind die im Finanzund Kapitalmarktrecht herausgebildeten Compliance-Pflichten, die oftmals Vorbild-, zumindest Ausstrahlungsbedeutung für das allgemeine Gesellschaftsrecht haben. Daher lohnt es sich, einen Blick auf die Entwicklung in diesen Bereichen zu werfen, zumal gerade das Finanzaufsichtsrecht wohl dasjenige Rechtsgebiet ist, das die längste Tradition in der Verrechtlichung von Unternehmensorganisationspflichten aufweist, aber auch am intensivsten in die inneren Strukturen der Unternehmen eingreift: ${ }^{18}$

\section{Compliance im Finanz- und Kapitalmarktaufsichtsrecht}

Im Bereich des Finanzmarktaufsichtsrechts sind sowohl im Wertpapierhandelsrecht als auch im Bank- und Versicherungsaufsichtsrecht entsprechende verpflichtende Vorgaben für die Einrichtung einer Compliance-Funktion verankert:

\section{Spezialgesetzliche Vorgaben (WpHG)}

Die allgemeinen organisatorischen Anforderungen aus $\mathbb{3} 3 \mathrm{WpHG}$ umfassen in \33 Abs. 1 S. 2 Nr. 1, S. 3 WpHG die Verankerung einer Compliance-Funktion, was wiederum auf Art. 13 Abs. 2 der Richtlinie 2004/39/EG (MiFID) und Art. 6 der Richtlinie 2006/73/EG (Durchführungsrichtlinie MiFID) zurückgehtNäher ausgeformt werden diese Vorgaben durch die WpDVerOV, ${ }^{19}$ insbesondere $\mathbb{S} \mathbb{S} 12 \mathrm{f} .{ }^{20}$ In der Praxis spielen aber vor allem die von der BaFin verabschiedeten allgemeinen Mindestanforderungen an die Compliance-Funktion und die weiteren Verhaltens-, Organisations- und Transparenzpflichten nach $\mathbb{\$} \mathbb{S} 31 \mathrm{ff}$. WpHG für Wertpapierdienstleistungsunternehmen (MaComp) ${ }^{21}$ eine erhebliche Rolle, da sie für die Marktteilnehmer das Verständnis der Anforderungen des WpHG durch die BaFin und damit deren Verwaltungspraxis abbilden. Zwar erheben die Interpretationen der BaFin als Verwaltungsanweisungen ${ }^{22}$ selbst keinen Anspruch auf Vollständigkeit; ${ }^{23}$ auch

17 Zur früheren Entwicklung und der Verwendung von Organisationspflichten als sekundäre Verkehrspflichten in allen Rechtsbereichen G. Spindler, Unternehmensorganisationspflichten: Zivilrechtliche und öffentlich-rechtliche Regelungskonzepte, 2. Aufl., Göttingen 2011, passim.

18 Näher zu den früheren Anforderungen Spindler, Unternehmensorganisationspflichten (Fn. 17), S. $186 \mathrm{ff}$.

19 Verordnung zur Konkretisierung der Verhaltensregeln und Organisationsanforderungen für Wertpapierdienstleistungsunternehmen - Wertpapierdienstleistungs-Verhaltens- und Organisationsverordnung v. 20.7.2007, BGBl. I, S. 1432.

20 S. auch zur Ausgestaltung durch WpDVerOV und MaComp: R. Sethe, Erweiterung der bank- und kapitalmarktrechtlichen Organisationspflichten um Reporting-Systeme, ZBB 2008, S. 421 (427f.).

21 Rundschreiben Nr. 4/2010 (WA) - vom 7.6.2010, zuletzt geändert am 7.12.2012, abrufbar unter: http://www.bafin.de/SharedDocs/Veroeffentlichungen/DE/Rundschreiben/rs 1004 wa macomp.html, (Letzter Abruf: 10.7.2013).

22 K. Langenbucher, Vorstandshaftung und Legalitätspflicht in regulierten Branchen, ZBB 2013, S. 16 (17).

23 Siehe z.B. MaComp AT 1 Tz. 1, insbesondere Tz.4. 
herrscht weitgehend Einigkeit darüber, dass sie keine normkonkretisierende Wirkung haben können, ${ }^{24}$ sondern nur das Ermessen der BaFin im Sinne allgemeiner Verwaltungsvorschriften binden können. Ihre große Bedeutung für die Praxis und die durchaus anderen Gremien im Verwaltungsrecht zeigt sich jedoch allein schon in den langwierigen Konsultationen der BaFin, die durchaus mit dem Prozess der Verabschiedung einer Verordnung verglichen werden können. Schließlich hat auch die ESMA inzwischen Leitlinien für die Organisation bzw. Einrichtung einer Compliance-Funktion verabschiedet. ${ }^{25}$

\12 Abs. 1 WpDVerOV verlangt zunächst eine frühzeitige Erkennung von Risiken, einhergehend mit einer klaren Kompetenzzuweisung zur Aufgabenerfüllung. Die Aufgabe der Compliance-Funktion ist gem. $\mathbb{} 33$ Abs. 1 S. 2 Nr. 1, S. 3 WpHG iVm. \12 Abs. 3 WpDVerOV maßgeblich von einer Beratung des operativen Bereichs, sowie der Überwachung der implementierten Vorkehrungen zur Einhaltung der Gesetzeskonformität im Bereich des WpHG geprägt. Die Überwachungsaufgabe umfasst die Erstellung eines Risikoprofils durch die Prüfung und Bewertung von Risiken, ${ }^{26}$ sowie die Prüfung der durch die einzelnen Bereiche durchzuführenden Eigenkontrollen mit eigenen zusätzlichen Stichproben. Zudem soll die Geschäftsleitung auf noch zu implementierende Maßnahmen hingewiesen werden. ${ }^{27}$ Eine Abstimmung der Compliance-Funktion mit den anderen unternehmensinternen Kontrollfunktionen ist ferner erforderlich. ${ }^{28}$ Obwohl die operativen Bereiche bereits selbst zur Eigenkontrolle ihres Handelns angehalten sind, wird die zusätzliche, wenn auch nur stichprobenartige, Überwachung dieser Bereiche durch die Compliance-Funktion nahe gelegt. ${ }^{29}$ Dies betrifft insbesondere die Überwachung der nach $\mathbb{S} \int 31 \mathrm{ff}$. WpHG eingerichteten Maßnahmen. ${ }^{30}$ Die Beratungsaufgabe beinhaltet die Unterstützung des operativen Bereichs im täglichen Geschäft bei Compliance-relevanten Sachverhalten und sieht insbesondere die Durchführung von regelmäßigen Schulungen der Mitarbeiter zu den an sie gestellten gesetzlichen Anforderungen vor. ${ }^{31}$

Neben diesen eher an Aufgaben und Funktionen orientierten Präzisierungen werden vor allem hinsichtlich der Aufbauorganisation Anforderungen an die betroffenen Unternehmen gestellt, wobei sich die konkrete Ausgestaltung nach den Besonder-

24 S. Niermann, Die Compliance-Organisation im Zeitalter der MaComp - eine Analyse ausgewählter Fragen, ZBB 2010, S. 400 (405 ff.); I. Koller, in: H.-D. Assmann/ U.H. Schneider (Hrsg.), Wertpapierhandelsgesetz, 6. Aufl., Köln 2012, \$33 Rn. 2.

25 Leitlinien zu einigen Aspekten der MiFID - Anforderungen an die Compliance Funktion vom 25. Juni 2012, ESMA/2012/388, abrufbar unter http://www.esma.europa.eu/system/files/2012-388_de.pdf.

26 MaComp BT 1.2.1.1.

27 MaComp BT 1.2.1.2.

28 MaComp BT 1.2.1.2 Tz. 7.

29 MaComp AT 6 Tz. 2.

30 MaComp AT 6 Tz. 3.

31 MaComp BT 1.2.3. 
heiten des Unternehmens richten soll. ${ }^{32}$ Unter anderem hat sich das Unternehmen

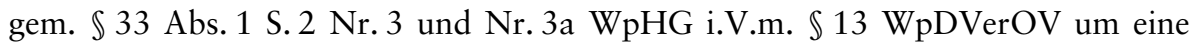
Vermeidung von Interessenkonflikten und Beeinträchtigungen der Kundeninteressen zu bemühen, ${ }^{33}$ was insbesondere dadurch erreicht werden kann, dass die Aufbauund Ablauforganisation klare Zuständigkeiten und Unabhängigkeiten („Chinese Walls“) im Umgang mit Compliance-relevanten Informationen beschreibt. ${ }^{34}$ Die Einrichtung und Unterhaltung der Compliance-Funktion steht grundsätzlich in der Gesamtverantwortung der Geschäftsleitung, kann aber auch einem Ressort zugewiesen werden. ${ }^{35}$ Eine externe Delegation (Outsourcing) ist unter Beibehaltung der Gesamtverantwortung bei der Geschäftsleitung unter den Voraussetzungen der $\mathbb{} 33$

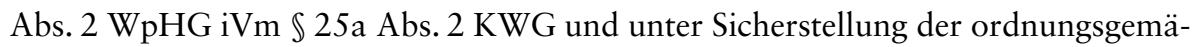
ßen Aufgabendurchführung möglich. ${ }^{36}$ Insgesamt soll eine „Compliance-Kultur“ angeregt werden, durch die der Anlegerschutz gestärkt und der notwendige Rahmen zur Bearbeitung von Compliance-Sachverhalten errichtet wird. ${ }^{37}$

Um der Compliance-Funktion die Durchführung ihrer Aufgaben zu ermöglichen, ist sie frühzeitig auf den relevanten Ebenen im geschäftlichen Bereich, sowie insbesondere bei der Korrespondenz mit den Aufsichtsbehörden einzubinden ${ }^{38}$ und mit ausreichenden personellen und sachlichen Mitteln auszustatten. ${ }^{39}$ Damit die Einbindung der Funktion Wirksamkeit entfaltet, sind die mit der Compliance-Funktion betrauten Mitarbeiter mit umfassenden Befugnissen auszustatten, insbesondere ist ihnen der Zugang zu den Compliance-relevanten Daten zu gewähren. ${ }^{40}$

Ähnlich den in vielfacher Hinsicht schon bekannten Beauftragten, wie Datenschutzbeauftragter ${ }^{41}$ etc., muss gem. $\mathbb{} 12$ Abs. 4 WpDVerOV die Unternehmensleitung einen Compliance-Beauftragten ernennen. ${ }^{42}$ Dieser muss im Sinne des $\mathbb{\$} 34 \mathrm{~d}$ Abs. 3 S. 2 WpHG die notwendige Zuverlässigkeit und Sachkunde besitzen, um seiner Aufgabe gerecht zu werden. ${ }^{43}$ Für den Compliance-Beauftragten ist ein ausreichend qualifizierter Vertreter zu bestellen, der zur Absicherung der ununterbrochenen Auf-

32 MaComp AT 3.2, BT 1; N. Wiederholt/A. Walter, Compliance - Anforderungen an die Unternehmensorganisationspflichten, BB 2011, S. 968 (969).

33 MaComp AT 6.2 Tz.1.

34 MaComp AT 6.2 Tz.3.

35 MaComp BT 1.1 Tz.1, Tz.2.

36 MaComp BT 1.3.4; MaRisk (BA) AT 9.

37 MaComp BT 1.1 Tz. 5.

38 MaComp BT 1.2.4.

39 MaComp BT 1.3.1.1.

40 MaComp BT 1.3.1.2.

41 Zur langen Geschichte der unterschiedlichen Beauftragten s. E. Rehbinder, Andere Organe der Unternehmensverfassung, ZGR 1989, S. 305 (307ff.); Spindler, Unternehmensorganisationpflichten (Fn. 17), S. 41 ff. (118 ff.) (265 ff.) ( 283 ff.).

42 MaComp BT 1.1 Tz.3; Wiederholt/Walter, Compliance (Fn. 32), S. 972.

43 MaComp BT 1.3.1.4. 
gabendurchführung dient. ${ }^{44}$ Nach $\mathbb{} 12$ Abs. 4 S. 3 (mit der Ausnahme in Abs. 5) WpDVerOV darf die mit der Compliance-Funktion betraute Personen nicht aktiv im operativen Geschäft tätig werden, ${ }^{45}$ um Interessenkollisionen auszuschließen. Zudem ist die fehlende Beeinflussung der mit der Compliance-Funktion betrauten Mitarbeiter sicherzustellen. ${ }^{46}$ Organisatorisch kann die Compliance-Funktion unter anderem mit dem Risikocontrolling oder der Rechtsabteilung verbunden werden, solange die Unabhängigkeit erhalten bleibt. ${ }^{47}$ Keine Verbindung darf jedoch mit der internen Revision vorgenommen werden, da diese von der Compliance-Funktion zu überwachen ist. ${ }^{48}$ Schließlich folgt aus $\mathbb{3} 33$ Abs. 1 S. 1 WpHG, dass die Vorgaben des $\mathbb{2} 25 \mathrm{a}$ Abs. 1 und Abs. 4 KWG, inklusive den MaRisk-Regelungen Anwendung finden. ${ }^{49}$

\section{Kreditinstitute}

Nicht nur Wertpapierdienstleistungsunternehmen ( $\$ 2$ Abs. 4 WpHG) unterfallen der Pflicht zur Einrichtung einer Compliance-Organisation, sondern auch Kreditinstitute als Teil des durch $\mathbb{\$} 25 \mathrm{a}$ Abs. 1 S. 3 Nr. 1 KWG geforderten internen Kontrollsystems. ${ }^{50}$ Das CRD IV-Umsetzungsgesetz ${ }^{51}$ listet nunmehr in $\$ 25$ a Abs. 1 S. 3 Nr. 3 c) KWG-E erstmals die Compliance-Funktion als Teil des internen Kontrollsystems explizit auf. Laut Gesetzesbegründung sollen Risiken aus der Nichtbefolgung rechtlicher Vorgaben entgegengewirkt werden, indem die Compliance-Funktion iSd. $\$ 25 a$ Abs. 1 S. 3 Nr. 3 c) KWG-E die Geschäftsleitung bei der Ausgestaltung entsprechender Maßnahmen unterstützt und zudem die Maßnahmen an sich bewertet und deren Qualität und Angemessenheit sichert und überwacht. ${ }^{52}$ Diese Umsetzung beruht auf den Vorgaben der EBA Guidelines on Internal Governance (GL

44 MaComp BT 1.3.2 Tz.2.

45 MaComp BT 1.1.1., BT 1.3.3.1; M. Kort, Rechtsfragen der Compliance-Organisation von Unternehmen außerhalb spezialgesetzlich geregelter Branchen im deutschen Recht, in: H. Altmeppen/H. Fitz/ H. Honsell (Hrsg.), Festschrift für Günther H. Roth zum 70. Geburtstag, 1. Aufl., München 2011, S. 407, (417 ff.).

46 MaComp BT 1.3.3 Tz.1.

47 MaComp BT 1.3.3.2 Tz.1, BT 1.3.3.3 Tz.1.

48 MaComp BT 1.3.3.2 Tz. 2.

49 MaComp AT 7.

50 MaComp AT 7 Tz. 2; MaRisk (BA) AT 4.3 Tz. 1; P. O. Mülbert, Bankenaufsicht und Corporate Governance - Neue Organisationsanforderungen im Finanzdienstleistungsbereich, BKR 2006, 349, 358; D. Weber-Rey, Gesellschafts- und aufsichtsrechtliche Herausforderungen an die Unternehmensorganisation, AG 2008, 345, 348 ff.; hierzu auch Wundenberg, Compliance und die prinzipiengeleitete Aufsicht über Bankengruppen, Tübingen 2012, S. $106 \mathrm{f}$.

51 Gesetz zur Umsetzung der Richtlinie 2013/36/EU über den Zugang zur Tätigkeit von Kreditinstituten und die Beaufsichtigung von Kreditinstituten und Wertpapierfirmen und zur Anpassung des Aufsichtsrechts an die Verordnung (EU) Nr. 575/2013 über die Aufsichtsanforderungen an Kreditinstitute und Wertpapierfirmen (CRD IV-Umsetzungsgesetz) vom 28.08.2013 BT-Drucks. 17/10974 (elektr. Vorabfassung), geändert durch BR-Drucks. 374/13 vom 17.05.13 und BR-Drucks. 544/13 vom 27.06.13, Inkrafttreten am 1.1.14.

52 Begr. RegE BT-Drucks. 17/10974 S. 108 (elektr. Vorabfassung). 
44). ${ }^{5354}$ Ursprünglich sahen Vorschläge des Gesetzes zur Abschirmung von Risiken und zur Planung der Sanierung und Abwicklung von Kreditinstituten und Finanzgruppen gem. $\$ 54$ a KWG-E sogar eigene Strafbestimmungen für die Verletzung von Risk- und damit auch Compliance-Managementsystemvorgaben vor; 55 inzwischen werden gem. $\$ 54 \mathrm{a}$ Abs. $3 \mathrm{KWG-E} \mathrm{iVm.}$ S 25c Abs. 4c KWG-E nur Verletzungen von Anordnungen der BaFin zur Konkretisierung unter Strafe gestellt. ${ }^{56}$

Zuvor fanden sich Konkretisierungen „nur“ in der MaRisk ${ }^{57}$ dergestalt, dass die Einhaltung der Normen überwacht und Risiken beurteilt werden müssen. ${ }^{58}$ Insbesondere soll sie die Geschäftsleitung beraten und durch einzurichtende Vorkehrungen zur Überwachung und Kontrolle die Entstehung von Risiken aus der Nichtbeachtung von Normen präventiv entgegenwirken. ${ }^{59}$ Allerdings ergeben sich viele Überlappungen mit den von der MaComp geforderten Compliance-Funktionen: So muss die Compliance-Funktion nicht notwendigerweise als abgetrennte Funktion eingerichtet werden, sondern kann auch anderen Kontrollsystemen angegliedert werden, wobei sie primär der Geschäftsleitung verantwortlich ist und ihr auch zu berichten hat. ${ }^{60}$ Die Berichterstattung an die Geschäftsleitung hat auf jeden Fall jährlich und bei anlassbezogenen Vorfällen zu erfolgen und muss auch vorzuschlagende Maßnahmen beinhalten, sofern Mängel in den implementierten Einrichtungen zur Risikoüberwachung festgestellt wurden. ${ }^{61}$

Auch für Kreditinstitute ist ein verantwortlicher Compliance-Beauftragter zu bestellen, der je nach Unternehmenszuschnitt auch ein Geschäftsleiter sein kann. ${ }^{62}$ Ebenso wie in der MaComp wird aber auch das Outsourcing der Funktion gestattet, sofern die Gesamtverantwortung bei der Geschäftsleitung verbleibt und die ordnungsgemäße Durchführung der Aufgaben dadurch nicht beeinträchtigt wird. ${ }^{63}$ Den mit der

53 EBA Guidelines on Internal Governance (GL 44), 27. Sept. 2011, III Title II Nr. 28, abrufbar unter: http://www.eba.europa.eu/cebs/media/Publications/Standards\%20and\%20Guidelines/2011/EBABS-2011-116-final-\%28EBA-Guidelines-on-Internal-Governance\%29-\%282\%29_1.pdf (zuletzt abgerufen am 31.5.2013).

54 Begr. RegE BT-Drucks. 17/10974 S. 108 (elektr. Vorabfassung).

55 Entwurf eines Gesetzes zur Abschirmung von Risiken und zur Planung der Sanierung und Abwicklung von Kreditinstituten und Finanzgruppen vom 4.3.2013 BT-Drucks. 17/12601 S. 28 f.; sehr kritisch Strafrechtsausschuss des Deutschen Anwaltvereins, Stellungnahme zum strafrechtlichen Teil des „Entwurfs eines Gesetzes zur Abschirmung von Risiken und zur Planung der Sanierung und Abwicklung von Kreditinstituten und Finanzgruppen“ (BT-Dr.17/12601), NZG 2013, S. 577 (580 ff.).

56 BR-Drucks. 378/13 S. 20 f.

57 Rundschreiben BaFin Nr. 18/2005 (BA), zuletzt geändert durch Rundschreiben BaFin Nr. 10/2012 (BA) vom 14.12.2012, abrufbar unter: http://www.bafin.de/SharedDocs/Veroeffentlichungen/DE/ Rundschreiben/rs_1210_marisk_ba.html?nn=2819248 (zuletzt abgerufen am 10.7.2013).

58 MaRisk (BA) AT 4.4.2.

59 MaRisk (BA) AT 4.4.2 Tz. 1.

60 MaRisk (BA) AT 4.4.2 Tz. 3.

61 MaRisk (BA) AT 4.4.2 Tz. 6.

62 MaRisk (BA) AT 4.4.2 Tz. 4.

63 MaRisk (BA) AT 9 Tz. 4. 
Compliance-Funktion betrauten Mitarbeitern muss ein uneingeschränkter Zugang zu den Compliance-relevanten Daten gewährt werden, damit sie ihre Aufgaben ordnungsgemäß erfüllen können. ${ }^{64}$

\section{Versicherungsunternehmen}

Last but not least verlangt auch das Versicherungsaufsichtsrecht im Gleichklang mit den übrigen finanzmarktrechtlichen Regelungen nach $\mathbb{S} 64 \mathrm{a}$ Abs. 1 S. 1, S. 2 iVm \ 7a Abs. 1 S. 4 VAG eine Compliance-Organisation, ${ }^{65}$ die bereits der Umsetzung der Solvency II-Richtlinie dienen soll. ${ }^{66}$ Durch die 10. VAG-Novelle ${ }^{67}$ wird die Compliance-Funktion ferner ausdrücklich als Teil des internen Kontrollsystems in $\mathbb{} 29$ Abs. 1 VAG-E gesetzlich vorgeschrieben. Nach $\$ 29$ Abs. 2 VAG-E wird die Compliance-Funktion neben der Beratung des Vorstands über die gesetzeskonforme Aufgabenerledigung im Unternehmen auch eine Risikoidentifizierung und -bewertung zur Aufgabe haben.

Anders als für das WpHG und das KWG sieht die MaRisk(VA) von 2009 bislang allerdings keine weiteren Konkretisierungen vor (was allerdings aufgrund der Novellierung in Bälde zu erwarten ist). Daher verwundert es nicht, dass bislang in der Diskussion in der Regel die parallelen Regelungen in den anderen Rechtsgebieten herangezogen werden. Die Anforderungen der Ausgestaltung haben sich an den Umständen des jeweiligen Unternehmens zu orientieren. ${ }^{68}$ Andererseits hat der Vorstand die Compliance in Gesamtverantwortung auszuführen, sodass eine vollständige Delegation der Verantwortlichkeit auch hier nicht möglich ist. ${ }^{69}$ Insbesondere bei kleineren Versicherungsunternehmen ist organisatorisch nicht unbedingt eine Compliance-Abteilung zu fordern, da diese ebenso gut in die Rechtsabteilung integriert wer-

64 MaRisk (BA) AT 4.4.2 Tz. 5.

65 Begr. RegE BT-Drucks. 16/6518 S. 17; Weber-Rey, Unternehmensorganisation (Fn. 50), S. 345 (356); M. Dreher, Das Risikomanagement nach $\ 64$ a VAG und Solvency II, VersR 2008, S. 998 (1004 ff.); J. Bürkle, Die rechtlichen Auswirkungen der MaRisk VA auf die Geschäftsorganisation von Versicherungsunternehmen, VersR 2009, S. 866 (869f.); Reese/Ronge, Versicherungsunternehmen (Fn. 5), S. 1220; Louven/Raapke, Aktuelle Entwicklungen in der Corporate Governance von Versicherungsunternehmen, VersR 2012, S. 257 (261); Hemeling, Organisationspflichten des Vorstands zwischen Rechtspflicht und Opportunität, ZHR 175 (2011), S. 368 (371); D. Wilm, Die ordnungsgemäße Geschäftsorganisation einer Versicherung, in: S. Hutter/ T. Baums (Hrsg.), Gedächtnisschrift für Michael Gruson, Berlin 2009, S. 465 (468) (473 f.)

66 Art. 46 der Richtlinie 2009/138/EG des Europäischen Parlaments und des Rates vom 25. November 2009 betreffend die Aufnahme und Ausübung der Versicherungs- und der Rückversicherungstätigkeit (Solvabilität II), ABl. L 335/1.

67 Entwurf eines Zehnten Gesetzes zur Änderung des Versicherungsaufsichtsgesetzes vom 18.4.2012 BTDrucks. 17/09342.

68 Begr. RegE BT-Drucks. 16/6518 S. 16 f.; MaRisk (VA) 4.1; P. Hemeling, Compliance im Erst- und Rückversicherungsunternehmen, CCZ 2010, S. 21 (22 f.); Louven/Raapke, Versicherungsunternehmen (Fn. 65), S. 257 (261); D. Wilm, Die ordnungsgemäße Geschäftsorganisation einer Versicherung, in: S. Hutter/ T. Baums (Hrsg.),,GS Gruson (Fn. 65), S. 465 (468).

69 Begr. RegE BT-Drucks. 16/6518 S. 16 f.; Bürkle, MaRisk VA (Fn. 65), S. 866 (870 f.); Reese/Ronge, Versicherungsunternehmen (Fn. 5), S. 1220; D. Wilm, Die ordnungsgemäße Geschäftsorganisation einer Versicherung, in: S. Hutter/ T. Baums (Hrsg.), GS Gruson (Fn. 65), S. 465 (473 f.). 
den kann, solange sie vom allgemeinen Risikomanagement abgegrenzt ist. ${ }^{70}$ Die Aufgaben der Compliance-Funktion bestehen auch hier im Wesentlichen in der Überwachung, Kontrolle der Verfahrensvorgänge und der Beratung der Mitarbeiter. ${ }^{71}$ Neben der Benennung eines Compliance-Beauftragten und der Einrichtung von direkten Berichtswegen zur Geschäftsleitung ist auf die Zuweisung hinreichender Kompetenzen zur Informationseinholung zu achten, damit eine unabhängige Aufgabenwahrnehmung erfolgen kann. ${ }^{72}$

\section{IDW-Prüfstandards}

Neben diesen soweit ersichtlich einzigen gesetzlichen (und untergesetzlichen) Normierungen zur Compliance gibt es natürlich eine Vielzahl von betriebswirtschaftlich orientierten Normierungen und Versuchen zur Standardsetzung, denen in der Praxis eine mehr oder minder große Bedeutung zukommt. ${ }^{73}$ Unter diesen ragt der nicht nur auf den Finanzsektor beschränkte Prüfstandard des Instituts der Wirtschaftsprüfer IDW PS 980 für das Compliance Management System (CMS) hervor. ${ }^{74}$ Dieser kommt bei der Prüfung des Compliance Management Systems durch einen Wirtschaftsprüfer zur Anwendung, sofern er nicht durch einen spezielleren IDW Prüfstandard verdrängt wird. ${ }^{75}$ Dass Gesellschaften, die der Wirtschaftsprüfung unterliegen, tendenziell diesen Standard anwenden werden, liegt auf der Hand, wenn sie nähere Auseinandersetzungen mit ihrem Abschlussprüfer vermeiden wollen. Rechtlich gesehen stellt der IDW Prüfstandard nur Grundsätze einer freiwilligen Prüfung auf und richtet sich als Berufsauffassung primär an die Wirtschaftsprüfer, ${ }^{76}$ sodass

70 Dreher, Risikomanagement (Fn. 65), S. 998 (1004); Hemeling, Compliance (Fn. 68), S. 21 (23); Reese/ Ronge, Versicherungsunternehmen (Fn. 5), S. 1232; Louven/Raapke, Versicherungsunternehmen, (Fn. 65), S. 257 (261).

71 Hemeling, Compliance (Fn.68), S. 21 (22); Reese/Ronge, Versicherungsunternehmen (Fn. 5), S. $1221 \mathrm{ff}$.

72 Hemeling, Compliance (Fn. 68), S. 21 (23 ff.).

73 Als Beispiel kann hier der „TR CMS 101:2011, Standard für Compliance Management Systeme (CMS)“ des TÜV Rheinland genannt werden, abrufbar unter: http://www.tuv.com/media/germany/ 60_systeme/compliance/compliance_standard_tr.pdf (zuletzt abgerufen am 10.7.2013).

74 IDW Prüfungsstandard: Grundsätze ordnungsmäßiger Prüfung von Compliance Management Systemen (IDW PS 980) vom 11.3.2011, abgedruckt in WPg Supplement 2/2011, S. 78 ff.; hierzu L. Böttcher, Compliance: Der IDW PS 980 - Keine Lösung für alle (Haftungs-)Fälle!, NZG 2011, S. 1054 (1054ff.); K. Wolf, Der IDW Prüfungsstandard 980 zur ordnungsmäßigen Prüfung von Compliance Management Systemen, DStR 2011, S. 997 (997 ff.); den IDW PS 980 als „Orientierungshilfe“ sehend A. Schemmel/ A. Minkoff, Die Bedeutung des Wirtschaftsstrafrechts für Compliance Management Systeme und Prüfungen nach dem IDW PS 980, CCZ 2012, S. 49 (53); zum Entwurf siehe B. Görtz, Der neue Compliance-Prüfungsstandard (EPS 980). Inhalte und Aussagen, CCZ 2010, S. 127 ff.; $H$. F. Gelhausen/ A. Wermelt, Haftungsrechtliche Bedeutung des IDW EPS 980: Grundsätze ordnungsmäßiger Prüfung von Compliance-Management-Systemen, CCZ 2010, S. 208 ff.; Rieder/Jerg, Überprüfung (Fn. 8), S. 201 ff.; J. Liese/ M. Schulz, Risikomanagement durch Compliance-Audits, BB 2011, S. 1347 (1350 f.).

75 IDW PS 980, WPg Supplement 2/2011, S. 78 (79) Tz. 2.

76 IDW PS 980, WPg Supplement 2/2011, S. 78 (79) Tz. 1; Görtz, EPS 980 (Fn. 74), S. 127; D. Eisolt, Prüfung von Compliance-Management-Systemen: erste Überlegungen zu IDW EPS 980, BB 2010, S. 1843. 
er sich davon maßgeblich in der Wirkungsweise von den gesetzlichen Aufsichtsbestimmungen und deren Interpretationen durch die BaFin unterscheidet. Seine faktische Wirkung ist indes im Rahmen der Abschlussprüfung unbestritten. Demgegenüber hat sich bislang noch kein ISO-Standard herausgebildet, der sich sonst für die verschiedensten Managementaufgaben bis hin zur Corporate Social Responsibility in der ISO $26000 \mathrm{ff}$. findet. ${ }^{77}$

Der Prüfungsstandard IDW PS 980 wurde vor allem von dem sog. COSO-Modell geprägt. ${ }^{78} \mathrm{Zu}$ beachten ist der Vorrang des IDW PS $340^{79}$ für die Prüfung des Risikofrüherkennungssystems nach $\mathbb{} 317$ Abs. 4 HGB, der als spezieller Standard die Anwendung des IDW PS 980 ausschließt. ${ }^{80}$ Jedoch zielt auch der IDW PS 980 auf ein effektives Compliance-System ab, sodass aus dessen Kernelementen Rückschlüsse gezogen werden können, welche Elemente ein solches System zu umfassen hat. Bereits der Prüfstandard PS $521^{81}$ zur Prüfung der Compliance-Funktion im Rahmen des Wertpapierdienstleistungsgeschäfts nach $\$ 36$ Abs. 1 Satz $1 \mathrm{WpHG}$ erwähnt für Wertpapierdienstleistungsunternehmen die Vorgaben zur Einrichtung und Unterhaltung einer unabhängigen und dauerhaften Compliance-Funktion, ${ }^{82}$ ohne jedoch aufgrund der Verweise in die Regelungen des $\mathbb{3} 3$ Abs. 1 Nr. 1 und 2 WpHG und \12 Abs. 3 WpDVerOV neue Anforderungen an diese Funktion aufzustellen. Durch die Bereitstellung der drei Prüfungsarten der Wirksamkeitsprüfung, ${ }^{83}$ Konzeptionsprüfung ${ }^{84}$ und Angemessenheitsprüfung ${ }^{85}$ werden durch den PS 980 den Auftraggebern der Prüfung Möglichkeiten an die Hand gegeben, um entweder eine umfassende Prüfung des CMS zu beauftragen (Wirksamkeitsprüfung) oder zunächst nur eine unabhängige Beurteilung des Entwicklungsstands des CMS (Konzeptions- oder An-

77 Zum ISO 26000, siehe G. Spindler, Corporate Social Responsibility in der AG - Mythos oder Realität?, in: B. Erle/W. Goette/D. Kleindiek et al. (Hrsg.), Festschrift für Peter Hommelhoff zum 70. Geburtstag, Köln 2012, S. 1133 (1136).

78 J. Schindler/ J. Haußer, Die Pflicht gesetzlicher Vertreter von Kapitalgesellschaften zur Aufdeckung von Unregelmäßigkeiten und die Reaktion des gesetzlichen Abschlussprüfers, WPg 2012, S. 233 (235 f.)

79 IDW Prüfungsstandard: Die Prüfung des Risikofrüherkennungssystems nach $\$ 317$ Abs. 4 HGB (IDW PS 340) vom 25.6.1999, abgedruckt in WPg 1999, S. 658 ff.

80 IDW PS 980, WPg Supplement 2/2011, S. 78 (79) Tz. 2 (87) Tz. A2; zum Entwurf Eisolt, Prüfung (Fn. 76), S. 1843 (1844).


WpHG (IDW PS 521) vom 6.3.2009, abgedruckt in WPg Supplement 2/2009, S. 14 ff.

82 IDW PS 521 WPg Supplement 2/2009, S. 14 (24) Tz. 61.

83 IDW PS 980, WPg Supplement 2/2011, S. 78 (80) Tz. 14.

84 IDW PS 980, WPg Supplement 2/2011, S. 78 (80) Tz. 16.

85 IDW PS 980, WPg Supplement 2/2011, S. 78 (80) Tz. 17. 
gemessenheitsprüfung) zu erhalten. ${ }^{86}$ Prüfungsgegenstand ist die vom Vorstand zu vertretende Beschreibung des Compliance Management Systems. ${ }^{87}$ Mit sieben Elementen gibt der IDW PS 980 Anknüpfungspunkte vor, an denen sich die Ausgestaltung des Compliance-Managementsystems ausrichten kann. ${ }^{88}$ Das System sollte erstens die Vorbildfunktion in den Hierarchie-Ebenen deutlich machen (ComplianceKultur). ${ }^{89}$ Damit Risiken erfasst werden können, müssen zunächst die mit dem System verfolgten Absichten geklärt sein (Compliance-Ziele). ${ }^{90}$ Nach Festlegung der Compliance-Ziele (policy) als zweites Element können darauf aufbauend drittens die Risiken identifiziert und bewertet werden (Compliance-Risiken). ${ }^{91}$ Sodann können aufgrund dieser Ergebnisse viertens Prozesse zur Prävention der festgestellten Risiken implementiert werden (Compliance-Programm). ${ }^{92}$ Daneben muss fünftens ein ordnungsgemäßer Rahmen mit festen Zuständigkeiten und genügender Mittelausstattung geschaffen werden (Compliance-Organisation). ${ }^{93}$ Es muss sechstens sichergestellt werden, dass die Informationen über die getroffenen Maßnahmen zu allen Hierarchieebenen durchdringen (Compliance-Kommunikation). ${ }^{94}$ Eine einmalige Befassung mit der Ausgestaltung des Systems reicht schließlich nicht aus, sodass siebtens eine stetige Überprüfung der implementierten Maßnahmen zu erfolgen hat (Compliance-Überwachung und Verbesserung). ${ }^{95}$

86 IDW PS 980, WPg Supplement 2/2011, S. 78 (80) Tz. 15; noch zum Entwurf: Görtz, EPS 980 (Fn. 74), S. 127 (129) (133); Gelhausen/Wermelt, Haftung (Fn. 74), S. 208 (211 f.); Eichler, Darstellung der vom IDW entwickelten Grundsätze ordnungsgemäßer Prüfung von Compliance-Management-Systemen, in: J. Wieland/ A. Schack (Hrsg.), Soziale Marktwirtschaft: Verantwortungsvoll gestalten, Frankfurter Allgemeine Buch, S. 197 (207f.); von Busekist/Hein, Mindestanforderungen (Fn. 8), S. 42; kritisch. Rieder/Jerg, Überprüfung (Fn. 8), S. 206.

87 IDW PS 980, WPg Supplement 2/2011, S. 78 (80) Tz. 12 f.; zum Entwurf: Görtz, EPS 980 (Fn. 74), S. 127 (128); Eisolt, Prüfung (Fn. 76), S. 1843 (1845).

88 U. Bergmoser, Integration von Compliance-Management-Systemen, BB Beil. 2010 Nr. 004, S. 2 (3); zum Entwurf: Görtz, EPS 980 (Fn. 74), S. 127 (130 f.); Eisolt, Prüfung (Fn. 76), S. 1843 (1845 ff.); von Busekist/Hein, Mindestanforderungen (Fn. 8), S. 41 f.; dazu auch S. Goslar, in: H-U.Wilsing (Hrsg.), Deutscher Corporate Governance Kodex Kommentar, München 2012, 4.1.3 DCGK Rn. 16.

89 IDW PS 980, WPg Supplement 2/2011, S. 78 (81) Tz. 23 (89f.) Tz. A14; hierzu von Busekist/Hein, Mindestanforderungen (Fn. 8), S. 45 f. mit dem Hinweis, dass dies auch aus $\mathbb{1 3 0}$ Abs. 1 OwiG folge; zum Entwurf: Görtz, EPS 980 (Fn. 74), S. 127 (130); Eisolt, Prüfung (Fn. 76), S. 1843 (1845).

90 IDW PS 980, WPg Supplement 2/2011, S. 78 (81) Tz. 23 (90) Tz. A15; von Busekist/Hein, Mindestanforderungen (Fn. 8), S. 46 f.; zum Entwurf: Görtz, EPS 980 (Fn. 74), S. 127 (130); Eisolt, Prüfung (Fn. 76), S. 1843 (1845 f.).

91 IDW PS 980, WPg Supplement 2/2011, S. 78 (81) Tz. 23 (90) Tz. A16; von Busekist/Hein, Mindestanforderungen (Fn. 8), S. 48; zum Entwurf: Görtz, EPS 980 (Fn. 74), S. 127 (130 f.); Eisolt, Prüfung (Fn. 76), S. 1843 (1846).

92 IDW PS 980, WPg Supplement 2/2011, 78, 81 Tz. 23, 90 f. Tz. A17; zum Entwurf: Görtz, EPS 980 (Fn. 74), S. 127 (131); Eisolt, Prüfung (Fn. 76), S. 1843 (1846).

93 IDW PS 980, WPg Supplement 2/2011, S. 78 (81) Tz. 23 (91) Tz. A18; zum Entwurf: Görtz, EPS 980 (Fn. 74), S. 127 (130); Eisolt, Prüfung (Fn. 76), S. 1843 (1846).

94 IDW PS 980, WPg Supplement 2/2011, S. 78 (82) Tz. 23 (91) Tz. A19; zum Entwurf: Görtz, EPS 980 (Fn. 74), S. 127 (131); Eisolt, Prüfung (Fn. 76), S. 1843 (1846).

95 IDW PS 980, WPg Supplement 2/2011, S. 78 (82) Tz. 23 (91f.) Tz. A20; zum Entwurf: Görtz, EPS 980 (Fn. 74), S. 127 (131); Eisolt, Prüfung (Fn. 76), S. 1843 (1846). 
In der Ausgestaltung gibt der Prüfstandard damit zwar auch nur wie die aufsichtsrechtlichen Vorgaben eine an das jeweilige Unternehmen anzupassende Struktur vor. ${ }^{96}$ Doch nimmt der PS 980 durch den Prüfnachweis, eine ermessensfehlerfreie Systemgestaltung erbracht zu haben, für sich mittelbar in Anspruch, ein umfassendes Compliance-Management darzustellen. ${ }^{97}$ Der Prüfstandard will aber kein über die gesetzlichen Vorgaben und insbesondere über die Interpretationen der BaFin hinausgehendes System hinaus aufstellen; insbesondere verweist der PS 980 auf die MaComp,${ }^{98}$ gleiches gilt für die Hinweise in anderen Prüfstandards wie dem PS 521 für die Prüfung der Compliance-Funktion im Rahmen des Wertpapierdienstleistungsgeschäfts nach $\mathbb{3} 36$ Abs. 1 Satz $1 \mathrm{WpHG}^{99}$ und PS 525 für die Prüfung des Risikomanagements von Kreditinstituten. ${ }^{100}$ Abweichungen zwischen den Prüfungsstandards und den behördlichen Interpretationen zeigen sich jedoch durchaus in der inhaltlichen Ausgestaltung. So enthält die Beschreibung der „Compliance-Kultur“ im IDW PS 980 ein Element, das nicht zum internen Kontrollsystem iSd. IDW PS 261 gehört. ${ }^{101}$

\section{E. Folgerungen für die AG}

Aufgrund der Vorgaben für Versicherungs- und Kreditwirtschaftsunternehmen stellt sich die Frage, ob diese Pflicht zur Einrichtung einer Compliance-Organisation nicht nur hinsichtlich des „Ob“, sondern auch des „Wie“ für das gesamte Aktienrecht und gegebenenfalls auch andere Unternehmen - gelten kann. ${ }^{102}$ So wird gerade von Praktikern sowohl den aufsichtsrechtlichen Regelungen als auch den IDW-Standards eine maßgebliche Ausstrahlung auf das allgemeine Aktienrecht zugesprochen. ${ }^{103}$

Gerade die sektorspezifischen Regulierungen, die bislang nicht für andere Branchen übernommen wurden, sprechen indes dafür, dass die tiefgehenden und detaillierten aufsichtsrechtlichen Bestimmungen keinesfalls eins zu eins übertragen werden können. ${ }^{104}$ Lediglich Anhaltspunkte können aus diesen Regulierungen gewonnen werden, welche bei der Ausgestaltung von Compliance-Systemen herangezogen werden

96 IDW PS 980, WPg Supplement 2/2011, S. 78 (79) Tz. 1.

97 IDW PS 980, WPg Supplement 2/2011, S. 78 (79) Tz. 1; anders Goslar (Fn. 88), 4.1.3 DCGK Rn. 17 ("rudimentär").

98 IDW PS 980, WPg Supplement 2/2011, S. 78 (87) Tz. A2 mit Fn 11.

99 IDW PS 521 WPg Supplement 2/2009, 14, 24 Tz. 59, mit einer Weiterverweisung auf die MaRisk (BA).

100 Explizit IDW Prüfungsstandard: Die Beurteilung des Risikomanagements von Kreditinstituten im Rahmen der Abschlussprüfung (IDW PS 525) vom 26.6.2010, WPg Supplement 3/2010, S. 4 (5) Fn. 5.

101 Bergmoser, Integration (Fn. 88), S. 2 (3).

102 Dreher, Aufsichtsrecht (Fn. 7), S. 519.

103 Für den IDW PS 340: Bergmoser/Theusinger/Gushurst, Corporate Compliance (Fn. 5), S. 4; für das Aufsichtsrecht: $J$. Rodewald, Gesetzestreue als Organisationsproblem, Compliance richtig managen, in: Maschmann (Hrsg.), Corporate Compliance und Arbeitsrecht, Baden-Baden 2009, S. 39 (47).

104 Ähnlich Dreher, Aufsichtsrecht (Fn. 7), S. 520; i.E. ebenso für mehr Ausgestaltungsspielraum Bergmoser/Theusinger/Gushurst, Corporate Compliance (Fn. 5), S. 10 f. 
können, ${ }^{105}$ im Sinne der Business Judgement Rule ${ }^{106}$ auch vom Vorstand geprüft und erwogen, aber nicht unbedingt übernommen werden müssen. Daher ist auch gegenüber den zahlreichen Vorschlägen und Beiträgen zur Einführung und Umsetzung von Compliance-Organisationen Zurückhaltung angebracht. Insbesondere kann eine Rechtspflicht etwa für alle Unternehmen, gleich welcher Größe, Branche etc., ein umfassendes Compliance-System einzurichten nicht angenommen werden. ${ }^{107} \mathrm{Wie}-$ derum kommt es hier entscheidend auf die Größe, Struktur und Lage des Unternehmens, das Risikopotential der Märkte, auf dem das Unternehmen tätig ist, sowie die Art des Kapitalmarktzugangs an. ${ }^{108}$

Zwar besteht auch nach Ziffer 4.1.3. des Deutschen Corporate Governance Kodex ${ }^{109}$ eine Pflicht des Vorstands börsennotierter Unternehmen, für die Einhaltungen der gesetzlichen Bestimmungen und der unternehmensinternen Richtlinien zu sorgen und auf deren Beachtung durch die Konzernunternehmen hinzuwirken, wobei dies nunmehr ausdrücklich als „Compliance“ bezeichnet wird. Jedoch lässt sich hierdurch weder eine Pflicht zur Etablierung einer bestimmten Compliance-Organisation entnehmen, ${ }^{110}$ da aufgrund fehlender „Soll“-Formulierung die Kodexbestimmung lediglich Informationscharakter besitzt, ${ }^{111}$ noch lassen sich hieraus (abgesehen vom

105 Kort, Verhaltensstandardisierung (Fn. 11), S. 82 f.; Wiederholt/Walter, Compliance (Fn. 32), S. 970; Sänger, Whistleblowing (Fn. 5), S. 231.

106 Zum Einfluß der Business Judgement Rule s. unten F. I.

107 Kort (Fn. 9) $\$ 91$ Rn. 65; Fleischer (Fn. 9), $\$ 91$ Rn. 50; ders., Vorstandsverantwortlichkeit (Fn. 9) S. 299 f.; Goette, Organisationspflichten (Fn. 5), S. 388 (396f.); Hemeling, Organisationspflichten (Fn. 65), S. 368 (386); T. Kremer/ C. Klahold, Compliance-Programme in Industriekonzernen, ZGR 2010, S. 113 (119f.); J. Campos Nave/ S. Bonenberger, Korruptionsaffären, Corporate Compliance und Sofortmaßnahmen für den Krisenfall, BB 2008, S.734; Kort, Verhaltensstandardisierung (Fn. 11), S. 84; Hauschka, Corporate Compliance (Fn. 9), S. 461 (462); ders., Korruptionsbekämpfung (Fn. 9), S. 878; Bürkle, Corporate Compliance (Fn. 9), S. 568 ff.; Schindler/Haußer, Unregelmäßigkeiten (Fn. 78), S. 233 (235 f.); J. Reichert, Corporate Compliance und der Grundsatz der Verhältnismäßigkeit, in: G. Krieger/ M. Lutter/ K. Schmidt (hrsg.), Festschrift für Michael HoffmannBecking zum 70. Geburtstag, München 2013, S. 943 (951f.); Kort, Rechtsfragen der ComplianceOrganisation von Unternehmen außerhalb spezialgesetzlich geregelter Branchen im deutschen Recht, in: Altmeppen et al. (Hrsg.), FS Roth (Fn. 45), S. 409; Hauschka (Fn. 2), 11 Rn. 23; a.A. Schneider, Compliance (Fn. 11), S. 648 f.

108 C. Hauschka, Von Compliance zu Best Practice, ZRP 2006, S. 258 (261); W. Müller in: J. Semler/ M. Peltzer (Hrsg.), Arbeitshandbuch für Vorstandsmitglieder, München 2005, $\$ 8$ Rn. 90; Fleischer (Fn. 9), $\$ 91$ Rn. 51; ähnlich Verse,Legalitätskontrollpflicht (Fn. 5), S. 401; Schneider, Compliance (Fn. 5), S. 1325.

109 Fassung vom 13. Mai 2013.

110 Kort (Fn. 9) $\$ 91$ Rn. 66; H.-M. Ringleb in: H.-M. Ringleb/ T. Kremer/ M. Lutter/A. von Werder (Hrsg.), Kommentar zum Deutschen Corporate Governance Kodex, 4. Aufl., München 2010, Rn. 624; Hauschka, Korruptionsbekämpfung (Fn. 9), S. 878; Kort, Verhaltensstandardisierung (Fn. 11), S. 84; Koch, Unternehmensverbund (Fn. 8), S. 1020; a.A. Schneider, Compliance (Fn. 11), S. 648; zum Nutzen von IT-Compliance L. Lensdorf, IT-Compliance - Maßnahmen zur Reduzierung von Haftungsrisiken von IT-Verantwortlichen, CR 2007, S. 413 (414).

111 J. Koch, Unternehmensverbund (Fn. 8), S. 1020;. 
Empfehlungscharakter des DCGK) Rückschlüsse auf die Auslegung des $\mathbb{S} 91$ Abs. 2 AktG ziehen. ${ }^{112}$

Bei aller Vorsicht lassen sich einige mehr oder minder allgemeingültige Eckpunkte festhalten: So muss die Einrichtung einer Compliance-Organisation sich auf sämtliche Zweige des Unternehmens erstrecken, um Pflichtverletzungen durch Mitarbeiter zu verhindern; ${ }^{113}$ eine Ausnahme kann nur dann eingreifen, wenn ein Bereich des Unternehmens besonders risikoanfällig für Pflichtverletzungen ist, andere dagegen nicht. $\mathrm{Zu}$ den Pflichtverletzungen gehören auch wirtschaftlich sinnvolle Verstöße durch Mitarbeiter, ${ }^{114}$ das Verbot nützlicher Rechtsverletzungen gilt allgemein. ${ }^{115} \mathrm{Da}$ die Compliance-Funktion das Risikomanagement zu überwachen hat, müssen beide Bereiche voneinander getrennt sein, ${ }^{116}$ auch wenn sie nicht völlig überschneidungsfrei sein mögen. ${ }^{117}$

Weitgehende Freiheit genießt der Vorstand bei der Ausgestaltung der ComplianceOrganisation: Auch wenn diese grundsätzlich dem Vorstand obliegt, kann er einzelne oder alle Funktionen auf geeignete Mitarbeiter delegieren, zB auf eine ComplianceStelle zur Überwachung der Organisationspflichten oder einen Compliance-Beauf-

112 So auch Kort (Fn. 9) $\$ 91$ Rn. 66; Mertens/Cahn (Fn. 5), $\$ 91$ Rn. 20; J. Liese, Much Adoe About Nothing? oder: Ist der Vorstand einer Aktiengesellschaft verpflichtet, eine Compliance-Organisation zu implementieren?, BB 2008, Beil. 005, S. 17 (20); J. Koch, Unternehmensverbund (Fn. 8), S. 1020; unklar J. Preußner, Risikomanagement im Schnittpunkt von Bankaufsichtsrecht und Gesellschaftsrecht, NZG 2004, S. 57 (60); anders wohl Campos Nave/Bonenberger, Korruptionsaffären (Fn. 107), S. 734 (735), die zur Eingliederung des Compliance-Systems in die interne Revision auf Kort, Verhaltensstandardisierung (Fn. 11), S. 83 verweisen, der sich in diesem Punkt nur auf den DCGK bezieht.

113 Spindler, Vorstandspflichten (Fn. 9), Rn. 51 f.; Goette, Organisationspflichten (Fn. 5), S. 388 (391); im Ansatz Campos Nave/Bonenberger, Korruptionsaffären (Fn. 107), S. 734 (734 f.); zur Zielsetzung der Compliance Organisation T. Lampert (Fn. 2), $\mathbb{9}$ Rn. 2 ff. Besondere Bedeutung erlangt die Compliance-Organisation für Insider-Trading in Wertpapierunternehmen; auf die Bedeutung einer Eingrenzung auf bestandsgefährdende Verstöße hinweisend Dreher, Vorstandsverantwortung (Fn. 7), S. 174.

114 Schneider, Compliance (Fn. 5), S. 1323; Verse, Legalitätskontrollpflicht (Fn. 5), S. 406; Fleischer, Corporate Compliance (Fn. 5), S. 1; ders., Aktienrechtliche Legalitätspflicht und „nützliche“ Pflichtverletzungen von Vorstandsmitgliedern, ZIP 2005, S. 141 (145 ff.).

115 BGHSt 52, 323 - Siemens = ZIP 2008, S. 2315 = NStZ 2009, S. 95; BGH NJW 2010, S. 3458 = NZG 2010, S. 1190; H. Merkt, Managerhaftung im Finanzsektor: Status Quo und Reformbedarf, in: B. Erle/W. Goette/D. Kleindiek et al. (Hrsg.), Festschrift für Peter Hommelhoff zum 70. Geburtstag, Köln 2012, S. 711 (713); Verse, Legalitätskontrollpflicht (Fn. 5), S. 406.

116 Dreher (Fn. 70), S. 998 (1004); Weber-Rey, Unternehmensorganisation (Fn. 50), S. 345 (348); Dreher, Vorstandsverantwortung (Fn. 7), S. 172 (174).

117 Dreher, Vorstandsverantwortung (Fn. 7), S. 173 ff.; insofern zutreffend von einer „Verzahnung“ ausgehend Wundenberg, Compliance (Fn. 50), S. 8; Compliance System als Teil des Risikomanagements: Schindler/Haußer, Unregelmäßigkeiten (Fn. 78), S. 233 (235); Lösler, Verständnis (Fn. 9), S. 105; Rodewald, Gesetzestreue als Organisationsproblem, Compliance richtig managen, in: Maschmann (Hrsg.), Compliance (Fn. 103), S. 34; hierzu auch Spindler, Bankengruppe (Fn. 7), S. 906 f. 
tragten. ${ }^{118}$ Dies mag bei größeren Unternehmen sicherlich sinnvoll ${ }^{119}$ und bei entsprechenden Risiken auch rechtlich geboten sein, nicht aber bei kleineren Gesellschaften mit kleiner organisatorischer Leitungsspanne. Sowohl die horizontale Delegation durch Aufgabenzuweisungen zu einzelnen Ressorts, als auch die vertikale Delegation mittels Auswahl von untergeordneten Personen stellen bei der Compliance-Organisation zulässige Mittel dar. ${ }^{120}$ In beiden Fällen ändert sich die Verantwortlichkeit der delegierenden Vorstandsmitglieder in eine Kontrollpflicht, die je nach Anlass intensiver oder geringer ausfallen kann. ${ }^{121}$

Eine Weisungsunabhängigkeit, wie sie von der BaFin im Finanzbereich verlangt wird, sowie uneingeschränkte Auskunfts-, Zugangs- und Einsichtsrechte sind dagegen nicht zwingend, außer bei besonders hohen Risiken, die eine vollkommen unabhängige Beurteilung erlauben. Auch hier bleibt die Verantwortung bei der Geschäftsleitung. ${ }^{122}$ Ebenso wenig muss stets eine Whistleblower-Hotline zur Unterstützung der Compliance-Funktion eingerichtet werden; sie mag einen sinnvollen Beitrag zur Erkennung von Verstößen leisten, ist aber grundsätzlich nicht zwingend. ${ }^{123}$ Ferner kann nicht davon ausgegangen werden, dass stets und generell ein Compliance Audit

118 Spindler, Vorstandspflichten (Fn. 9), Rn. 52; U. H. Schneider/S. H. Schneider, Konzern-Compliance als Aufgabe der Konzernleitung, ZIP 2007, S. 2061 (2065); Fleischer (Fn. 9), \91 Rn. 58; Mertens/ Cahn (Fn. 5), $\$ 91$ Rn. 36; Kremer/Klahold, Industriekonzerne (Fn. 107), S. 113 (118) (125); anders wohl Kort (Fn. 9) \$91 Rn.67; Ringleb (Fn. 110), Rn. 628 ff.; Dreher, Vorstandsverantwortung (Fn. 7), S. 172; Kort, Rechtsfragen der Compliance-Organisation von Unternehmen außerhalb spezialgesetzlich geregelter Branchen im deutschen Recht, in: Altmeppen et al. (Hrsg.), FS Roth (Fn. 45), S. 410; Wiederholt/Walter, Compliance (Fn. 32), S.972; a.A. Schneider, Compliance (Fn. 11), S. $649 \mathrm{f}$.

119 So Kremer/Klahold, Industriekonzerne (Fn. 107), S. 113 (123 ff.); zum Handbuch Wiederholt/Walter, Compliance (Fn. 32), S. 970.

120 Goette, Organisationspflichten (Fn. 5), S. 388 (394f.); Fleischer, Kartellrechtsverstöße (Fn. 5), S. 1072; Liese, Compliance-Organisation (Fn. 112), S. 17 (21); Meier-Greve, Vorstandshaftung (Fn. 5), S. 2556; zum Versicherungsunternehmen M. Dreher/ M. Schaaf, Versicherungsunternehmensrecht und Risikomanagement, WM 2008, S. 1765 (1769); Reese/Ronge, Versicherungsunternehmen (Fn. 5), S. 1221; bzgl. vertikaler Delegation Kort, Rechtsfragen der Compliance-Organisation von Unternehmen außerhalb spezialgesetzlich geregelter Branchen im deutschen Recht, in: Altmeppen et al. (Hrsg.), FS Roth (Fn. 45), S. 409.

121 Goette, Organisationspflichten (Fn. 5), S. 388 (395); Fleischer, Corporate Compliance (Fn. 5), S. 2 mwN.; bzgl. vertikaler Delegation Kort, Rechtsfragen der Compliance-Organisation von Unternehmen außerhalb spezialgesetzlich geregelter Branchen im deutschen Recht, in: Altmeppen et al. (Hrsg.), FS Roth (Fn. 45), S. 409.

122 Spindler, Vorstandspflichten (Fn. 9), Rn. 52.

123 Kremer/Klahold, Industriekonzerne (Fn. 107), S. 113 (133f.); M. Passarge, Risiken und Chancen mangelhafter Compliance in der Unternehmensinsolvenz, NZI 2009, S. 86 (87); K.-D. Bussmann/S. Matschke, Die Zukunft der unternehmerischen Haftung bei Compliance-Verstößen, CCZ 2009, S. 132 (135); Kort, Rechtsfragen der Compliance-Organisation von Unternehmen außerhalb spezialgesetzlich geregelter Branchen im deutschen Recht, in: Altmeppen et al. (Hrsg.), FS Roth (Fn. 45), S. 412; anders wohl K. Korte, Die Information des Aufsichtsrats durch die Mitarbeiter, Frankfurt am Main u.a. 2009, S. 143; zum Whistle-Blowing aus individualarbeitsrechtlicher Sicht M. Kort, Individualarbeitsrechtliche Fragen des Whistleblowing, in: G. Hönn/ H. Oetker/ T. Raab (Hrsg.), Festschrift für Peter Kreutz zum 70. Geburtstag, München 2010, S. 247 ff.; zur Ausgestaltung aus Arbeitnehmersicht H.-J. Fritz, Whistleblowing - Denunziation oder Wettbewerbsvorteil? Inhalt und Grenzen des Whistleblowings im Rahmen von Corporate Compliance, in: Maschmann (Hrsg.), Compliance (Fn. 103), S. $111 \mathrm{ff.}$ 
durchzuführen ist; ${ }^{124}$ gleiches gilt für die Forderungen nach Durchführung eines unternehmensbezogenen Compliance Standard-Trainingsprogrammes oder die unternehmensbezogene Erstellung von Handbüchern. ${ }^{125}$

\section{F. Rechtsfolgen bei Verstoß, insbesondere Haftung.}

Die Welle an Compliance-Beratungen ist wie schon erwähnt keineswegs durch die besonderen gesetzlichen Festlegungen induziert, sondern entspringt auch dem Bedürfnis der Leitungsorgane, sich gegenüber dem Vorwurf eines etwaigen Organisationsverschuldens als Anknüpfungspunkt für straf- sowie zivilrechtliche Verantwortlichkeit bzw. Haftungsrisiken zu entlasten. Seitdem die Tendenz zu verzeichnen ist, die geschäftsführenden Organe für Verstöße ihres Unternehmens selbst in die Pflicht zu nehmen, etwa über die Figur von Garantenstellungen infolge Organisationshoheit, ${ }^{126}$ rückt die Frage vermehrt in den Vordergrund, wie sich das Organmitglied konkret entlasten kann. Ein „Königsweg“ aus der Verantwortlichkeit bzw. Haftung scheint gerade die Schaffung einer Compliance-Organisation zu sein.

\section{Haftung nach §93 Abs. 1 AktG, insbesondere Business Judgement Rule}

Aus gesellschaftsrechtlicher Sicht steht die Pflichtwidrigkeit nach $\mathbb{9} 93$ Abs. 1 AktG im Vordergrund. Entsprechend den allgemeinen Prinzipien ${ }^{127}$ obliegt die Compliance-Organisation als Ausprägung der allgemeinen Pflicht, für die Vermeidung von Rechtsverstößen zu sorgen, als Aufgabe dem gesamten Organ Vorstand, wobei nicht ressortzuständige Vorstandsmitglieder nur Überwachungspflichten einzuhalten haben. ${ }^{128}$ Allerdings kann bei regulierten Branchen wie der Versicherungsaufsicht die Verletzung der Pflichten zur Einrichtung einer Compliance auch als Abberufungsgrund für die Behörde in Betracht kommen. ${ }^{129}$

Entscheidend ist für die gesellschaftsrechtliche Haftung der Vorstandsmitglieder indes die Anwendung der Business Judgement Rule nach $\$ 93$ Abs. 1 S. 2 AktG. Demnach kann dem Vorstand bei unternehmerischen Entscheidungen keine Pflichtwid-

124 Zur Ausgestaltungsmöglichkeit eines Compliance-Audit, insbesondere auch rechtsvergleichend siehe Liese/Schulz, Compliance-Audits (Fn. 74), S. 1347.

125 Hierzu Schneider, Compliance (Fn. 11) S. 649; Hauschka, Reaktion (Fn. 9), S. 260; Passarge, Unternehmensinsolvenz (Fn.123), S. 86 (87); Lampert, Compliance-Organisation (Fn. 113), $\$ 9$ Rn 19 ff.; Wiederholt/Walter, Compliance (Fn. 32), S.970; Bergmoser, Integration (Fn. 88), S. 2 (4f.); Kort, Rechtsfragen der Compliance-Organisation von Unternehmen außerhalb spezialgesetzlich geregelter Branchen im deutschen Recht, in: Altmeppen et al. (Hrsg.), FS Roth (Fn. 45), S. 412.

126 Grundlegend für das Strafrecht BGHSt 37, 106 - Lederspray, für das Zivilrecht BGHZ 109, 297 Baustoff; zur Entwicklung Spindler, Unternehmensorganisationspflichten (Fn. 17), S. 877 (Strafrecht), S. 844 ff. (Zivilrecht); G. Spindler, in: H. G. Bamberger/ H. Roth, Kommentar zum Bürgerlichen Gesetzbuch, Bd. 2, 3. Aufl. 2012, München 2012, $\$ 823$ Rn. 271 ff. mwNachw.

127 S. Spindler, in: W. Goette / M. Habersack (Hrsg.), Münchener Kommentar zum Aktiengesetz, Bd. II, 4. Aufl., München 2013, $\mathbb{S} 93$ Rn. 149.

128 Bezogen auf $\mathbb{9} 91$ Abs. 2 auch VG Frankfurt aM WM 2004, S. 2157.

129 So für das Riskmanagement schon VG Frankfurt aM (Fn. 128), S. 2157. 
rigkeit vorgeworfen werden, wenn er Entscheidungen auf der Grundlage angemessener Informationen und Bewertungen trifft, um damit dem unternehmerischen Risiko seiner Tätigkeit Rechnung zu tragen. ${ }^{130}$ Auch im Bereich der Organisationspflicht kommt der Vorstand in den Genuss der Business Judgement Rule nach $\mathbb{\$} 93$ Abs. 1 S. 2 AktG, da die Art und Weise der Einrichtung eines Risikomanagementsystems, insbesondere auch der Compliance-Organisation eine unternehmerische Entscheidung darstellt. ${ }^{131}$ Denn der Vorstand muss Kosten und Nutzen einer Organisation bzw. verschiedener Modelle abwägen, dabei auch Prognosen über Risiken, deren Entwicklung und Ausmaß treffen. Grundlegend für die Entlastung des Vorstandes ist wie auch sonst für $\mathbb{} 93$ Abs. 1 S. 2 AktG, dass sich der Vorstand mit den für die Einrichtung einer Compliance erhältlichen Informationen auseinandersetzt, wozu auch die Analyse der oben dargelegten besonders gesetzlich regulierten Organisationsformen gehört, ob diese für sein Unternehmen geeignet sind und ggf. wie er diese zu modifizieren hat - ohne dass er diese übernehmen müsste. Insbesondere die Analyse von rechtlichen Risiken muss in diesem Rahmen sorgsam durchgeführt werden. Insgesamt besteht zwar keine allgemeine Pflicht zur Einrichtung; doch muss der Vorstand prüfen, ob und wie er ein Compliance-System einrichtet. ${ }^{132}$ Dabei ist selbstverständlich stets die „gelebte“ Organisation entscheidend; entgegen einer oft gebräuchlichen Praxis genügt allein das Führen eines „Compliance-Handbuchs“, das die Compliance-Vorkehrungen im Unternehmen hinreichend beschreibt, ${ }^{133}$ für sich genommen nicht, um den Vorstand von seiner Pflicht zu entlasten, wenn die tatsächliche Organisation sich davon entfernt hat - „Papier ist geduldig“.

Eine völlig andere Frage bezieht sich auf die Anerkennung einer Business Judgement Rule im Bereich von Rechtsbegriffen, wie sie gerade dem Aufsichtsrecht, wie erwähnt, zugrunde liegt (inzwischen auch gerne „Legal Judgement Rule“ genannt). ${ }^{134}$ Denn das Finanzmarkt-, Bank- und Versicherungsaufsichtsrecht erlegt den Unternehmen zwingend die dargelegten Organisationspflichten auf, garniert mit zahlreichen unbestimmten Rechtsbegriffen, die den Rechtsanwender häufig vor die Frage stellen, welche Organisation denn nun als ,angemessen“ zu gelten hat; unter Zugrundelegung der klassischen öffentlich-rechtlichen Maßstäbe müsste der Vorstand in vollem Umfang einer gerichtlichen Nachprüfung bei Anwendung dieser Rechtsbegriffe unterliegen - was im Widerspruch zu der sonst im Gesellschaftsrecht

130 Näher dazu Spindler (Fn. 127), $\$ 93$ Rn. 36 ff. mwNachw.

131 Ebenso Bergmoser, Integration (Fn. 88), S. 2 (3); von Busekist/Hein, Mindestanforderungen (Fn. 8), S. $44 \mathrm{f}$. weisen auf die Einschränkungen des Auswahlermessens durch die Vorgaben des $\$ 130$ Abs. 1 OWiG hin.

132 Fleischer, Corporate Compliance (Fn. 5), S. 3; ders., Kartellrechtsverstöße (Fn. 5), S. 1070 (1072); Kremer/Klahold, Industriekonzerne (Fn. 107), S. 113 (120).

133 Bergmoser/Theusinger/Gushurst, Grundlagen (Fn. 5), S.9; Kort, Verhaltensstandardisierung (Fn. 11), S. 85.

134 S. etwa J. Bürkle, Aufsichtsrechtliches Legal Judgment: Sachlicher Anwendungsbereich und prozedurale Voraussetzungen,VersR 2013, S. 792 ff. mwNachw. 
anerkannten Business Judgement Rule stünde. ${ }^{135}$ Indes ist schon für das Gesellschaftsrecht die Erstreckung der Business Judgement Rule auf unbestimmte Rechtsbegriffe dargelegt worden, ${ }^{136}$ was inzwischen auch für das Aufsichtsrecht Gefolgschaft gefunden hat. Allerdings bedarf dies noch einer weiteren Diskussion, die hier aus Raumgründen nicht geführt werden kann, da das Aufsichtsrecht gerade aufgrund der öffentlich-rechtlichen Grundlage anderen Prinzipien unterliegt als das Gesellschaftsrecht; eine einfache Akzessorietät der Business Judgement Rule zum Aufsichtsrecht als eine Art „safe harbor“ auch im Aufsichtsrecht kann daher nicht vollzogen werden und übersieht grundlegende Unterschiede zwischen haftungs- und gefahrenabwehrrechtlichen Ansätzen. ${ }^{137}$

Genauso relevant ist aber auch die umgekehrte Frage, ob der Vorstand sich auf bestimmte Risikomanagementmodelle verlassen kann. Die Antwort liegt angesichts der Anforderungen nach $\mathbb{9} 93$ Abs. 1 S. 2 AktG auf der Hand: Eine angemessene Informationsgrundlage bedingt stets eine nähere Auseinandersetzung mit der Situation des eigenen Unternehmens und dessen Risiken; ${ }^{138}$ ein blindes Vertrauen auf ein bestimmtes Modell oder dessen ungeprüfte Übernahme scheiden damit von vornherein aus. Gerade die bei Organisationen erforderliche Einzelfallanpassung einerseits und die Abstraktionshöhe von Risikomanagementmodellen kann keine automatische Entlastungswirkung hinsichtlich der Sorgfaltsanforderungen nach $\mathbb{9} 93$ begründen, wenn der Vorstand bestimmte Modelle einhält; dies gilt auch für die Prüfstandards des IDW. Denn die Prüfung stellt gerade bezogen auf Managementsysteme lediglich eine Systemprüfung dar, da die Eignung der dadurch veranlassten Entscheidungen nicht in die Prüfung miteinbezogen wird. ${ }^{139}$ Die Prüfung dient lediglich dem Zweck, dass mögliche Mängel im System aufgedeckt werden, die in der Folge behoben werden können; ${ }^{140}$ es handelt sich damit im Wesentlichen um eine innere Konsistenz-

135 Bürkle, Legal Judgment (Fn. 134), S. 792 (799 ff.); zuvor C. Armbrüster, Haftung der Geschäftsleiter bei Verstößen gegen $\$ 64$ a VAG, VersR 2009, S. 1293 (1296).

136 G. Spindler, Die Haftung von Vorstand und Aufsichtsrat für fehlerhafte Auslegung von Rechtsbegriffen, in: A. Heldrich et al. (Hrsg.), FS Canaris (Fn. 4), S. 403 (415 ff.); darauf aufbauend C. Thole, Managerhaftung für Gesetzesverstöße - Die Legalitätspflicht des Vorstands gegenüber seiner Aktiengesellschaft, ZHR 173 (2009), S. 504 (521); Langenbucher, Regulierte Branchen (Fn. 22), S. 16 alle mwNachw.

137 Eingehend zum nötigen Spielraum im Öffentlichen Recht bei Organisationspflichten, insbesondere bei Verwaltungsakten, Vorgaben etc. Spindler, Unternehmensorganisationspflichten (Fn. 17), S. $558 \mathrm{ff}$., insbesondere S. $562 \mathrm{ff}$.

138 Anders offenbar P. Hommelhoff/ D. Mattheus, Gesetzliche Grundlagen: Deutschland und international, in: D. Dörner/ P. Horváth/ H. Kagermann (Hrsg.), Praxis des Risikomanagements, Stuttgart 2000, S. 13 f., die von einer Vermutungswirkung sprechen.

139 IDW PS 340, WPg 1999, S. 658 (658) Tz. 6 (660) Tz. 19; T. Klindt/ C. Pelz/I. Theusinger, Compliance im Spiegel der Rechtsprechung, NJW 2010, S. 2385 (2387) (zum IDW PS 980); G. Förschle/ C. Almeling, in: H. Ellrott/ G. Förschle et al. (Hrsg.), Beck'scher Bilanz-Kommentar, 8. Aufl., München 2012, $\$ 317$ Rn. 86.

140 Eisolt, Prüfung (Fn. 76), S. 1843 (zum IDW PS 980). 
prüfung, vergleichbar etwa den Qualitätsmanagementsystemprüfungen. ${ }^{141} \mathrm{Ob}$ zudem die Prüfer immer die nötige Rechtskunde besitzen oder nicht vielmehr nur die eigene Auffassung der Wirtschaftsprüfer reflektieren, ${ }^{142}$ wird aus der Praxis in Zweifel gezogen. ${ }^{143}$ Dies schließt nicht aus, dass die Beachtung der Prüfstandards oder von Risikomanagementmodellen als Indiz für die Einhaltung der Anforderungen des \93 Abs. 1 S. 2 AktG herangezogen werden können. ${ }^{144}$

Gerade für die Finanzbranche stellt sich die Frage, ob nicht darüber hinaus gehend das Vertrauen in die Konkretisierungen durch die BaFin den Vorstand auch vor einer gesellschaftsrechtlichen Haftung im Innenverhältnis schützt. Durch die Befolgung der BaFin-Standards soll eine ermessensfehlerfreie Ausübung der Organisationsspielräume vorliegen; nur bei Hinweisen auf ein fehlerhaftes Verständnis durch die BaFin soll der Vorstand gehalten sein, hiervon abzuweichen. ${ }^{145}$ Dem ist grundsätzlich zuzustimmen, da sich der Vorstand sonst unter Umständen in einer unauflöslichen Pflichtenkollision befindet; doch ist andererseits auch daran zu erinnern, dass die Rechtsprechung allgemein bislang öffentlich-rechtliche Vorgaben stets nur als Mindestanforderungen begriffen hat, über die im Einzelfall aber das Zivilrecht hinausgehen kann, wenn besondere Gefahrenlagen sich stellen. ${ }^{146} \mathrm{Nicht}$ viel anders stellt sich die Situation in der AG hinsichtlich der Haftung gegenüber der Gesellschaft dar: Auch hier ist ein blindes Vertrauen auf die Vorgaben der BaFin (oder anderer Behörden) nicht gerechtfertigt, vielmehr muss der Vorstand die Angemessenheit der Vorgaben für das eigene Unternehmen prüfen und unter Umständen modifizieren, wobei er gehalten ist, sich mit der BaFin ins Benehmen über Ausnahmen etc. zu setzen.

141 Siehe hierzu Spindler, Unternehmensorganisationspflichten (Fn. 17), S. 296 ff.

142 Eisolt, Prüfung (Fn. 76), S. 1843; W. Niemann, Grundsätze ordnungsmäßiger Durchführung von Abschlussprüfungen im Umbruch?, DStR 2003, S. 1454 (1455); Görtz, EPS 980 (Fn. 74), S. 127; Böttcher, IDW PS 980 (Fn. 74), S. 1054 (1057); K.-P. Naumann, in: IDW (Hrsg.), WP-Handbuch, Bd. I, 14. Aufl., Düsseldorf 2012, A 365.

143 Rieder/Jerg, Überprüfung (Fn. 8), S. 204; Böttcher, IDW PS 980 (Fn. 74), S. 1054 (1057); K. Moosmayer, Compliance: Praxisleitfaden für Unternehmen, 2. Aufl., München 2012, S. 91.

144 Gelhausen/Wermelt, Haftung (Fn. 74), S. 208 (210); K.-H.Withus/ O. Hein, Prüfung oder Zertifizierung eines Compliance Management Systems, Voraussetzungen und mögliche Rechtsfolgen, CCZ 2011, S. 125 (129 f.); von Busekist/Hein, Mindestanforderungen (Fn. 8), S. 42; a.A. Rieder/Jerg, Überprüfung (Fn. 8), S. 204 ff.; Böttcher, IDW PS 980 (Fn. 74), S. 1054 (1057); Moosmayer, Compliance (Fn. 143), S. 91 f.; unklar Schemmel/Minkoff, Wirtschaftsstrafrecht (Fn. 74), S. 49 ff.

145 Langenbucher, Regulierte Branchen (Fn. 22), S. 23.

146 BGHZ 139, 79, 83; 139, 43, 46 f.; BGH NJW 1999, S. 2364; C.-W. Canaris, Schutzgesetze - Verkehrspflichten - Schutzpflichten, in: C.-W. Canaris/U. Diedrichsen (Hrsg.), Festschrift für Karl Larenz zum 80. Geburtstag am 23. April 1983, München 1983, S.27 (55 f.); G. Spindler (Fn. 126), $\$ 823$ Rn. 251 mwN. 


\section{Haftung gegenüber Dritten und strafrechtliche Verantwortlichkeit}

Eine Außenhaftung kommt entsprechend den allgemeinen Regeln nur in engen Grenzen in Betracht. ${ }^{147}$ Die Verletzung von Organisationspflichten ist nicht per se haftungsbegründend, da sie nur sekundäre Pflichten sind, die sich stets auf eine primäre Verkehrspflicht oder den Schutz eines Rechtsgutes beziehen. ${ }^{148}$ Insbesondere kann \91 Abs. 2 AktG nicht als Schutzgesetz iSd. $\int 823$ Abs. 2 BGB qualifiziert werden. ${ }^{149}$ Demgegenüber kann die Verletzung der Compliance-Pflichten im Rahmen des Organisationsverschuldens erhebliche Bedeutung gewinnen - sofern man der Rechtsprechung zur zivilrechtlichen Außenhaftung von Organmitgliedern folgen mag. Voraussetzung ist hier stets, dass man überhaupt die Organe im Sinne von Garanten bzw. Verkehrssicherungspflichtigen gegenüber Dritten ansieht; erst dann kann überhaupt eine mangelhafte Compliance-Organisation sich als Verletzung von Verkehrssicherungspflichten gegenüber Dritten ergeben. Besonderheiten gerade der Compliance resultieren daraus aber nicht; vielmehr handelt es sich um das allgemeine Problem der Außenwirkung eines Organisationsverschuldens, das hier aus Raumgründen nicht vertieft werden kann.

Deutlich weitergehende Haftungsrisiken - auch strafrechtlicher Art - können sich aber für Vorstände ergeben, die auf Grund der Notierung ihres Unternehmens an einer US-Börse den Bestimmungen des Sarbanes-Oxley-Acts unterliegen, da das hiernach für alle Kapitalgesellschaften (und nicht nur den Bankensektor) geforderte interne Kontrollsystem weit über die nach deutschen Recht geforderten Maßnahmen hinausgeht. ${ }^{150}$

Darüber hinaus ist die Verletzung von Compliance-Pflichten wiederum im Rahmen der strafrechtlichen Garantenstellung der Geschäftsleitung von Relevanz. Dies hat

147 Näher Spindler, Vorstandspflichten (Fn. 9), Rn. 63; Spindler (Fn. 127) \$93 Rn. 298 ff.

148 Näher dazu G. Spindler, Unternehmensorganisationspflichten (Fn. 17), S. 781 ff.

149 So auch Fleischer (Fn. 9), $\$ 91$ Rn. 46; Mertens/Cahn (Fn. 5), $\$ 91$ Rn. 29; D. Bibr/ M. Kalinowsky, Risikofrüherkennungssystem bei nicht börsennotierten Aktiengesellschaften - Haftungsfalle für Vorstand, Aufsichtsrat und Wirtschaftsprüfer, DStR 2008, S. 620 (625); S. Blasche, Die Mindestanforderungen an ein Risikofrüherkennungs- und Überwachungssystem nach $\$ 91$ Abs. $2 \mathrm{AktG}, \mathrm{CCZ}$ 2009, S. 62 (63).

150 S. dazu M. Gruson/ M. Kubicek, Der Sarbanes-Oxley Act, Corporate Governance und das deutsche Aktienrecht (Teil II), AG 2003, S. 393 (396 ff.); G. Lanfermann/ S. Maul, Auswirkungen des Sarbanes-Oxley Acts in Deutschland, DB 2002, S. 1725 (1728 ff.); C. Kersting, Auswirkungen des Sarbanes-Oxley-Gesetzes in Deutschland - Können deutsche Unternehmen das Gesetz befolgen?, ZIP 2003, S. 233 (233 f.) (235 f.); M. Glaum/ D. Thomaschewski/ S. Weber, Die Vorschriften zur Einrichtung und Dokumentation eines internen Kontrollsystems nach Section 404 Sarbanes-Oxley Act: Umsetzung durch deutsche Unternehmen, KoR 2006, S. 206 ff.; T. Büssow/T. Taetzner, Sarbanes-Oxley Act Section 404: Internes Kontrollsystem zur Sicherstellung einer effektiven Finanzberichterstattung im Steuerbereich von Unternehmen - Pflicht oder Kür?, BB 2005, S. 2437 (2438ff.); K. Wolf, Zur Anforderung eines internen Kontroll- und Risikomanagementsystems im Hinblick auf den (Konzern-) Rechnungslegungsprozess gemäß BilMoG, DStR 2009, S. 920 (922 ff.); Spindler, Vorstandspflichten (Fn. 9), Rn. 63; G. Obermayr (Fn. 2), $\$ 17$ Rn. 20 ff.; ebenso zu den höheren Anforderungen der Compliance-Systeme im Dodd-Frank-Act T. Schürrle/F. Fleck, „Whistleblowing Unlimited” - Der U.S. Dodd-Frank-Act und die neuen Regeln der SEC zum Whistleblowing, CCZ 2011, S. 218 (221). 
der 5. Strafsenat in einem obiter dictum gerade für die Verantwortlichkeit eines Compliance-Officers deutlich vor Augen geführt, indem das Gericht grundsätzlich eine Garantenpflicht des Compliance-Officers zur Verhinderung von Straftaten aus dem Unternehmen heraus gegenüber Dritten annahm. ${ }^{151}$ Der BGH schloss in seinem obiter dictum für den Compliance-Officer aus den zivilrechtlich übernommenen Vertragspflichten auf eine dementsprechende strafrechtliche Garantenpflicht eines Compliance-Officers, ${ }^{152}$ sowie für den entscheidungserheblichen Fall des Innenrevisors von den konkreten Aufgaben des Dienstpostens auf eine dementsprechende Garantenpflicht. ${ }^{153}$ Die Aufgaben des Compliance-Officers sollen insbesondere die Prävention von Straftaten gegenüber Dritten umfassen. ${ }^{154}$ Die Garantenpflicht sei „die notwendige Kehrseite ihrer gegenüber der Unternehmensleitung übernommenen Pflicht, Rechtsverstöße und insbesondere Straftaten zu unterbinden “. ${ }^{155}$ Hieraus wird zum Teil der Rückschluss dahingehend gezogen, dass dem Vorstand ebenfalls eine Strafbarkeit wegen Unterlassens drohe, wenn dieser erkennt, dass es zu einer solchen Straftat kommen soll und trotzdem nicht gegen die Begehung einschreitet. 156

Wie schon generell zur Frage der Strafbarkeit aufgrund einer allgemeinen Garantenstellung des Vorstands infolge seiner Organisationspflichten (Lederspray-Fall etc.), ${ }^{157}$ gilt auch hier, dass bereits der Ausgangspunkt, aus den zivilrechtlichen Pflichten im Innenverhältnis (!) eine Garantenpflicht der Unternehmensleitung gegenüber außenstehenden Dritten abzuleiten, mehr als zweifelhaft ist. Darüber hinaus hinkt der Vergleich zwischen einem freiwillig eingerichteten Compliance-Officer und einem zwingend zu bestellenden umweltschutzrechtlichen Betriebsbeauftragten als Überwachungsgaranten ${ }^{158}$ dergestalt, da es für den „normalen “ Compliance-Officer außerhalb der Regelungen im Finanzsektor keine gesetzliche Bestimmung gibt, die den Tätigkeitsumfang verbindlich festlegt. ${ }^{159}$ Allein eine unzureichende Organisati-

151 BGHSt 54, 44, 50 Tz. 27.

152 BGHSt 54, 44, 50 Tz. 27 unter Verweis auf O. Kraft/ K. Winkler, Zur Garantenstellung des Compliance-Officers - Unterlassungsstrafbarkeit durch Organisationsmangel, CCZ 2009, S. 29 (32).

153 BGHSt 54, 44, 51 Tz.30.

154 BGHSt 54, 44, 49 f. Tz. 27 unter Verweis auf J. Bürkle (Fn. 2), $\$ 8$ Rn. 1 ff.

155 BGHSt 54, 44, 50 Tz. 27 unter Verweis auf Kraft/Winkler, Garantenstellung (Fn. 152), S. 29 (32); kritisch J. Campos Nave/ H. Vogel, Die erforderliche Veränderung von Corporate Compliance-Organisationen im Hinblick auf gestiegene Verantwortlichkeiten des Compliance Officers, BB 2009, S. 2546 (2547), die neben den arbeitsrechtlichen Sanktionen eine zusätzliche strafrechtliche Garantenpflicht für nicht erforderlich erachten.

156 Kremer/Klahold, Industriekonzerne (Fn. 107), S. 113 (140); Schneider, Compliance (Fn. 5), S. 1322; Fleischer (Fn. 9), $\$ 91$ Rn. 63; T. Behling, Compliance versus Fernmeldegeheimnis, BB 2010, S. 892 Fn. 5; T. Wybitul, Strafbarkeitsrisiken für Compliance-Verantwortliche, BB 2009, S. 2590 (2591 f.); R. Raum, Strafrechtliche Pflichten von Compliance-Beauftragten - Zum Urteil des Bundesgerichtshofs vom 17.7.2009 (5 StR 394/08) -, CCZ 2012, S. 197.

157 BGHSt 37, 106 - Lederspray = NJW 1990, S. 2560.

158 Kraft/Winkler, Garantenstellung (Fn. 152), S. 29 (32).

159 Campos Nave/Vogel,Veränderung (Fn. 155), S. 2546 (2548). 
on zur Verhinderung von Straftaten kann nicht zur Verletzung einer Garantenpflicht führen. ${ }^{160}$ Die Pflicht zur Verhinderung von schädigenden Handlungen besteht gegenüber der Gesellschaft, nicht gegenüber außenstehenden Dritten. ${ }^{161}$

\section{G. Konzernbezogene Compliance-Anforderungen}

\section{Konzernbezogene Organisationspflichten?}

Die wirtschaftliche Realität besteht aber nicht nur aus Einheitsunternehmen, die nur einen Rechtsträger aufweisen; vielmehr ist die berühmte Einheit in der Vielheit das Kennzeichen moderner Unternehmensgruppen, die von dezentraler bis äußerst zentraler Führung bei gleichzeitiger Vielschichtigkeit von Rechtsträgern im (wirtschaftlichen) Unternehmen oszillieren. Dementsprechend liegt die Frage nach einer konzernweiten Compliance nahe. Der Gesetzgeber lässt den Rechtsanwender (und -wissenschaftler) dabei weitgehend im Stich, da die Gesetzesbegründung zu $\mathbb{} 91$ Abs. 2 AktG sowohl Anhaltspunkte zur Gleichstellung des Einheitsunternehmens mit dem Konzern enthält als auch die unterschiedlichen gesellschaftsrechtlichen Möglichkeiten betont. ${ }^{162}$ Auch nach Ziff. 4.1.4 zusammen mit der Präambel des DCGK erstreckt sich das Risikomanagement und -controlling auf das Konzernunternehmen. ${ }^{163}$ Daher soll den Vorstand der Obergesellschaft einer Unternehmensverbindung die Pflicht treffen, ein konzernweites Früherkennungssystem zu schaffen, ${ }^{164}$ wobei Umfang und Intensität dieser Pflicht sich danach richte, in welcher Weise das herrschen-

160 Mertens/Cahn (Fn. 5), \$91 Rn. 36; S. Thomas, Strafrecht CCZ 2009, S. 239 (240); anders wohl Wybitul, Strafbarkeitsrisiken (Fn. 156), S. 2590 (2592); Schneider, Compliance (Fn. 5), S. 1322.

161 BGH NJW 2012, S. 3439 (3441) Tz. 22 f.

162 Begr. RegE BT-Drucks. 13/9712 S. 15; s. auch Hommelhoff/Mattheus, Gesetzliche Grundlagen: Deutschland und international, in: D. Dörner et al. (Hrsg.), Praxis (Fn. 138), S. 32; P. Hommelhoffl D. Mattheus, Risikomanagement im Konzern - ein Problemaufriß, BFuP 2000, S. 217 (219 ff.); M. Lutter, Konzernphilosophie vs. konzernweite Compliance und konzernweites Risikomanagement, in: M. Habersack/ P. Hommelhoff (Hrsg.), Festschrift für Wulf Goette zum 65. Geburtstag, München 2011, S. 289 (295 f.).

163 Ringleb (Fn. 110), Rn. 642; Goslar (Fn. 88), 4.1.4 DCGK Rn. 6; Fleischer (Fn. 9), $\$ 91$ Rn. 41; Mertens/Cahn (Fn. 5), $\$ 91$ Rn. 18.

164 Welf/Müller (Fn. 108) $\ 8$ Rn. 103; T. Liebscher, in: W. Müller/ T. Rödder (Hrsg.), Beck'sches Handbuch der AG, 2. Aufl., München 2009, $\$ 6$ Rn. 117; Hommelhoff/Mattheus, Risikomanagement (Fn. 162), S. 217 (222 ff.); J. Preußner/ F. Becker, Ausgestaltung von Risikomanagementsystemen durch die Geschäftsleitung, NZG 2002, S. 846; Löbbe, Unternehmenskontrolle im Konzern, Heidelberg 2003, S. 197 ff., insbes. S. 213 ff.; vgl. auch Arbeitskreis „Externe und Interne Überwachung der Unternehmung “ der Schmalenbach-Gesellschaft, DB 2000, Beil. 11 zu Heft 37, Tz. 15; wohl auch M. R. Theisen, Risikomanagement als Herausforderung für die Corporate Governance, BB 2003, S. 1426 (1429); ähnlich Müller-Michaels in: W. Hölters (Hrsg.), Aktiengesetz Kommentar,

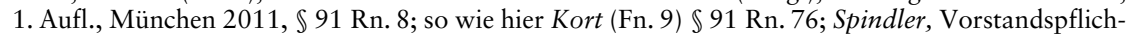
ten (Fn. 9), Rn. 62. 
de Unternehmen seine Konzernleitungsmacht im jeweiligen Fall ausübt; ${ }^{165}$ beim Beherrschungsvertrag etwa soll sie am stärksten ausgeprägt sein. ${ }^{166}$ Diese Pflicht soll Ausdruck der allgemeinen Konzernleitungspflicht sein. ${ }^{167}$

Diese konzernweite Erstreckung der an sich gesellschaftsrechtlichen Compliancepflicht findet ihren Widerhall inzwischen im Kartellrecht durch die Zurechnung von Pflichtverstößen der Tochtergesellschaft gegenüber der Muttergesellschaft, etwa in der Etex-Entscheidung des Bundeskartellamts auf der Grundlage von $\mathbb{\$} 130$ $\mathrm{OWiG}^{168}$ oder dem Urteil des EuGH in der Sache Akzo Nobel. ${ }^{169}$

Eine konzernweite Compliancepflicht berücksichtigt indes nicht hinreichend, dass der Konzern als solcher eben gerade nicht als Einheit geregelt wurde; ${ }^{170}$ ein unmittelbarer Einfluss der Obergesellschaft auf untergeordnete Gesellschaften ist nur unter ganz bestimmten Voraussetzungen, wie etwa $\$ 308$ AktG im Vertragskonzern, möglich. Schon der Prämisse, nämlich dass die Obergesellschaft eine umfassende Konzernleitungspflicht habe, kann indes nicht zugestimmt werden, ${ }^{171}$ weil sie eine Pflicht

165 Hommelhoff/Mattheus, Risikomanagement (Fn. 162), S. 217 (227); Preußner/Becker, Ausgestaltung (Fn. 164), S. 846 (847); H. Rieger, Gesetzeswortlaut und Rechtswirklichkeit im Aktiengesetz, in: M. Lutter/ M. Scholz/ W. Sigle (Hrsg.), Festschrift für Martin Peltzer zum 70. Geburtstag, Köln 2001, S. 339, 346; s. auch Fleischer (Fn.9), \$91 Rn. 41; ders., Konzernleitung und Leitungssorgfalt der Vorstandsmitglieder im Unternehmensverbund, DB 2005, S. 759 (764): Jdf. Verpflichtung für die Einrichtung einer konzerndimensionalen Risikoerfassung und -auswertung; Eckert: in T. Wachter (Hrsg.), Kommentar zum Aktiengesetz, 1. Aufl., Köln 2012, \91 Rn. 10; Müller-Michaels (Fn. 164) \91 Rn. 8.

166 Hommelhoff/Mattheus, Risikomanagement (Fn. 162), S. 217 (225 f.); Rieger, FS Peltzer (Fn. 165), 1999, S. 339 (346); Preußner/Becker, Ausgestaltung (Fn. 164), S. 846 (847).

167 U. H. Schneider/S. H. Schneider, Konzern-Compliance (Fn. 118), S. 2061 (2062 f.); Hommelhoffl Mattheus, Risikomanagement (Fn. 162), S. 217 (227) (zusammenfassend).

168 BKartA, Az. B1-200/06 vom 9.2.2009, abrufbar unter www.bundeskartellamt.de.

169 EuGH EuZW 2009, S. 816 - Akzo Nobel NV; dazu Schneider, Compliance (Fn. 5), S. 1324; Lutter, FS Goette (Fn. 162), 2011, S. 289 (290 f.); M. Habersack, Gedanken zur konzernweiten ComplianceVerantwortung des Geschäftsleiters eines herrschenden Unternehmens, in: S. Bechtold/ J. Jickeli/ M. Rohe (Hrsg.), Recht, Ordnung und Wettbewerb, Festschrift zum 70. Geburtstag von Wernhard Möschel 2011 Ort , S. 1175 (1177f.); Grundmeier, Rechtspflicht zur Compliance im Konzern, Köln 2012, S. 487 (490).

170 Ebenso M. Habersack, Gedanken zur konzernweiten Compliance-Verantwortung des Geschäftsleiters eines herrschenden Unternehmens, in: S. Bechtold et al. (Hrsg.), FS Möschel (Fn. 169), S. 1175 (1181f.).

171 Ebenso Fleischer, Corporate Compliance (Fn. 5), S. 3; ders. (Fn. 9), $\$ 76$ Rn. 86; Widmann, Das Risikomanagement als Funktionselement der Corporate Governance der Aktiengesellschaft, BadenBaden 2010, S. 73; Verse, Legalitätskontrollpflichten (Fn. 5), S. 407 f.; Goette, Organisationspflichten (Fn. 5), S. 388 (393). 
zur Verschaffung gesellschaftsrechtlicher Einflussrechte implizieren würde. ${ }^{172}$ Voraussetzung jeder Compliance ist die Beschaffung von Informationen, auf deren Grundlage eine Risikosteuerung erfolgen kann. Selbst hier ergeben sich jedoch bereits je nach Rechtsform der abhängigen Gesellschaft unter Umständen enge Grenzen, insbesondere bei der nur faktisch beherrschten AG im Sinne von $\ 311 \mathrm{ff}$. AktG. Der Vorstand kann hier nur dazu gehalten sein, solche Entwicklungen auf der Ebene der Muttergesellschaft (im Sinne einer Konzernrechnungslegung) zu erfassen, die bei einer Konsolidierung im Rahmen der Konzernbilanz ( $\$ 297$ Abs. 3 HGB) relevant sind. ${ }^{173}$ Risiken anderer Konzerngesellschaften sind dann iRd. gesellschaftsrechtlich zulässigen Informationsbeschaffung zu erfassen, wenn sie ein Risiko für die Muttergesellschaft darstellen können. ${ }^{174}$

Daraus ergibt sich ein abgestuftes Bild eines konzernweiten Risikomanagements: Ausgangspunkt ist die Vorstandspflicht der Muttergesellschaft zur ordentlichen Verwaltung des Beteiligungsbesitzes und der Verringerung daraus resultierender Risiken. ${ }^{175}$ Aus den untergeordneten Gesellschaften können sich Risiken ergeben, die auch Risiken für die Muttergesellschaft implizieren. ${ }^{176}$ Daraus folgt die Verpflichtung des Vorstands der Obergesellschaft, diejenigen Rechte zur Einflussnahme auf die Untergesellschaften wahrzunehmen, die zur Erfüllung des Pflichtenmaßstabs eines ordentlichen und gewissenhaften Geschäftsleiters notwendig sind, um die Interessen der eigenen Gesellschaft zu wahren. ${ }^{177}$ Die Intensität der Aufgabenwahrneh-

172 Spindler, Unternehmensorganisationspflichten (Fn. 17), S. 954 f.; Huth, Die Vorstandspflicht zur Risikoüberwachung, Baden-Baden 2006, S. 59; offen U. Hüffer, in: Hüffer (Hrsg.), Kommentar zum Aktiengesetz, 10. Aufl., München 2012, $\mathbb{S} 91$ Rn. 6. Eine Konzernleitungspflicht im Grundsatz auch ablehnend etwa Flume, Die juristische Person, Berlin u.a. 1983, S. 90 Fn. 97; H.-G. Koppensteiner in: W. Zöllner/ U. Noack, Kölner Kommentar zum Aktiengesetz, 3. Aufl., Köln u.a. 2004, $\$ 308$ Rn. 41; B. Kropff, Zur Konzernleitungspflicht, ZGR 1984, S. 112 (116 ff.); E. Rehbinder, Besprechung von Peter Hommelhoff, die Konzernleitungspflicht, ZHR 147 (1983), S. 464 (467 ff.); H. P. Westermann, Grundfragen der Rechtsfortbildung im Aktienkonzernrecht, in: P. Hofmann et al. (Hrsg.), Festschrift für Klemens Pleyer zum 65. Geburtstag, Köln 1986, S. 421 (426 ff.); Mertens/ Cahn (Fn. 5), $\mathbb{\int} 91$ Rn. 18; T. Bürgers/ A. Israel, in: T. Bürgers/ T. Körber (Hrsg.), Kommentar zum Aktiengesetz, 2. Aufl., Heidelberg 2011, $\$ 91$ Rn. 8; Müller-Michaels (Fn. 164) \91 Rn 8; ausführlich Spindler, Vorstandspflichten (Fn. 9), Rn. 62 ff.; zum Versicherungskonzern Dreher/Schaaf, Versicherungsunternehmensrecht (Fn. 120), S. 1765 (1772); die Konzernleitungspflicht in Bezug auf Compliance auch ablehnend T. Fett/ I. Theusinger, Compliance im Konzern - Rechtliche Grundlagen und praktische Umsetzung, BB Beil. 2010 Nr. 004, S. 6 (8).

173 Hüffer (Fn. 172), $\$ 91$ Rn. 6; Mertens/Cahn (Fn. 5), \$91 Rn. 18; Spindler, Vorstandspflichten (Fn. 9), Rn. 62.

174 M. Endres, Organisation der Unternehmensleitung aus der Sicht der Praxis, ZHR 163 (1999), S. 441 (451); Fleischer, Corporate Compliance (Fn. 5), S. 5; Schneider, Compliance (Fn. 5), S. 1324 ff.; Spindler, Bankengruppe (Fn. 7), S. 916; Verse, Legalitätskontrollpflichten (Fn. 5), S. 414 ff.

175 M. Habersack, Gedanken zur konzernweiten Compliance-Verantwortung des Geschäftsleiters eines herrschenden Unternehmens, in: S. Bechtold et al. (Hrsg.), FS Möschel (Fn. 169), S. 1175 (1182 f.).

176 Widmann, Risikomanagement (Fn. 171), 2010, S. 73.

177 Hüffer (Fn. 172), $\mathbb{S} 76$ Rn. 17 a; K. J. Hopt, in: K. J. Hopt/ H. Wiedemann (Hrsg.), Großkommentar zum Aktiengesetz, Bd. 3, 4. Aufl., Berlin 2008, $\mathbb{9} 93$ Rn. 114; Kort (Fn. 9), $\$ 76$ Rn. 140; K.-P. Martens, Die Organisation des Konzernvorstands, in: F. Kübler et al. (Hrsg.), Festschrift für Theodor Heinsius zum 65. Geburtstag am 25. September 1991, Berlin 1991, S. 523 (531f.). 
mung im Konzern hängt von den rechtlichen Einflussmöglichkeiten auf die untergeordneten Gesellschaften ab. ${ }^{178}$ Aufgrund des auch im Konzern bestehenden unternehmerischen Ermessens nach $\mathbb{\int} 93$ Abs. 1 S. 2 AktG relativiert sich diese Pflicht und ist selbst im Vertrags- und Eingliederungskonzern nicht als Pflicht zur umfassenden Leitung zu verstehen. ${ }^{179}$ Ein Weisungsrecht nach $\$ 308$ Abs. 1 AktG beinhaltet nicht zugleich eine Weisungspflicht, ${ }^{180}$ sodass selbst im Vertragskonzern der Vorstand nicht zur zentralen Leitung verpflichtet ist. ${ }^{181}$

\section{Konzernbezogene Compliance}

Dies gilt auch für die Frage einer Compliance-Organisation im Konzern: Auch hier soll nach einer verbreiteten Auffassung die Compliance-Organisation für sämtliche Unternehmen des Konzerns gelten, allerdings im Rahmen eines weiten Ermessensspielraums. ${ }^{182}$ Der Vorstand der Muttergesellschaft soll verpflichtet sein, für eine konzernweite Überwachung zu sorgen, die auch die Sicherstellung rechtmäßigen Verhaltens in den Konzerngesellschaften umfasst. ${ }^{183}$ Dies entspricht Ziff. 4.1.3 des DCGK, wonach der Vorstand auf die Beachtung der Gesetzes- und Unternehmensrichtlinieneinhaltung durch die Konzernunternehmen hinzuwirken habe - obgleich dem kein Empfehlungscharakter im Sinne eines Comply-or-Explain von der Kommission beigemessen wurde. ${ }^{184}$ In Kreditinstitutsgruppen bedarf es hingegen aufgrund aufsichtsrechtlicher Vorgaben in $\$ 25$ a Abs. 1 S. 1, Abs. 1a S. 1, Abs. 1b S. 1 KWG einer Compliance-Organisation, die sich auf alle Gruppenunternehmen erstreckt, allerdings nur bezüglich der Absicherung der Finanzstabilität, sodass auch hier keine Organisation zur umfassenden Normeneinhaltung gefordert wird. ${ }^{185}$

178 Fleischer (Fn. 9), $\mathbb{} 76$ Rn. 91.

$179 \operatorname{Kort}($ Fn. 9), $\mathbb{S} 76$ Rn. 144 f.; Fleischer (Fn. 9), $\$ 76$ Rn. 88; Hüffer (Fn. 172), \$ 76 Rn. 17 a; Schneider, Compliance (Fn. 5), S. 1326.

180 Emmerich, in: V. Emmerich/ M. Habersack (Hrsg.), Kommentar zum Aktien- und GmbH-Konzernrecht, 7. Aufl., München 2013, 308 AktG Rn. 34; Fleischer (Fn.9), $\$ 76$ Rn. 87; D. Kleindiek, Konzernstrukturen und Corporate Governance: Leitung und Überwachung im dezentral organisierten Unternehmensverbund, in: P. Hommelhoff/ K. J. Hopt/ A. v. Werder, Handbuch Corporate Governance, Köln Stuttgart 2003, S. 571 (579); Lutter, FS Goette (Fn. 162), 2011, S. 289 Fn. 1 mwN.

181 Rieger, FS Peltzer (Fn. 165), 2001, S. 339 (346); Koppensteiner (Fn. 172), \$ 308 Rn. 60; Emmerich (Fn. 180) $\$ 308$ AktG Rn. 34; G. Krieger, in: M. Hoffmann-Becking (Hrsg.), Münchener Handbuch des Gesellschaftsrechts, Bd. IV, Aktiengesellschaft, 3. Aufl., München 2007, $\mathbb{S} 70$ Rn. 155.

182 Kremer/Klahold, Industriekonzerne (Fn. 107), S. 113 (117) (122) (142); Schneider, Compliance (Fn. 5), S. 1326; Lutter, FS Goette (Fn. 162), 2011, S. 289 (292); Bürkle (Fn. 2), \$ 8 Rn. 64 f.

183 U.H. Schneider, Die Wahrnehmung öffentlich-rechtlicher Pflichten durch den Geschäftsführer, in: M. Lutter/ P. Ulmer/ W. Zöllner (Hrsg.), Festschrift 100 Jahre GmbH-Gesetz, Köln 1992, S. 473 (489 ff.); Hopt (Fn. 177), $\mathbb{S} 93$ Rn 98; T. E. Abeltshauser, Leitungshaftung im Kapitalgesellschaftsrecht, Köln 1998, S. 45; Fleischer (Fn. 165), S. 759 (763); ders. Corporate Compliance (Fn. 5), S. 3 f.; Grundmeier, Konzern (Fn. 169), S. 487 (498f.).

184 Ringleb (Fn. 110), Rn. 616; Goslar (Fn. 88), 4.1.3 DCGK Rn. 14.

185 T. Tröger, Konzernverantwortung in der aufsichtsunterworfenen Finanzbranche, ZHR 177 (2013), S. 475 (492). 
Auch hier gilt indes, dass die konkrete Verantwortung für die Einrichtung und Ausgestaltung einer Compliance-Organisation bei den Tochtergesellschaften nicht bei der Muttergesellschaft liegt, sondern bei den nachgeordneten Gesellschaften, die nur in Ausübung der ihr obliegenden Aufgaben von der Muttergesellschaft überwacht werden, ${ }^{186}$ sofern dies ihr gesellschaftsrechtlich möglich ist. Aufgrund der fehlenden Verpflichtung zum Ausbau konzernweiter Einflussrechte kann es keine Pflicht geben, bei unzureichenden Möglichkeiten zur Durchsetzung einer konzernweiten Compliance-Organisation eine Auflösung der Konzernbeziehung bzw. gar eine Verschmelzung in Betracht zu ziehen. ${ }^{187}$ Überwachungspflichten des Vorstands der Obergesellschaft hinsichtlich der Normeneinhaltung durch die Tochter- und Enkelgesellschaften bedingen zum einen Möglichkeiten der entsprechenden unmittelbaren Informationsbeschaffung, zum anderen auch der Intervention auf der Ebene der nachgeordneten Gesellschaften. ${ }^{188}$ Die praktische Durchsetzung der Überwachung befindet sich dabei stets im Ermessen der jeweiligen Konzernobergesellschaft. ${ }^{189}$

Daher können konzernweite „compliance-audits“, insbesondere durch Stichproben bei den Untergesellschaften, ${ }^{190}$ keine zwingende Verpflichtung darstellen. Entsprechend eines Compliance-Beauftragten auf einzelgesellschaftlicher Ebene kann es sich einzelfallabhängig im Konzern anbieten, für die Konzern-Ebene ein vergleichbares Pendant zu bestellen ${ }^{191}$ - sofern die Obergesellschaft über entsprechende Einflussmöglichkeiten verfügt. Eine Verpflichtung des Vorstands der Obergesellschaft zur Einrichtung von konzernweiten Informations- und Berichtswegen, ${ }^{192}$ die sowohl re-

186 Goette, Organisationspflichten (Fn. 5), S. 393; G. Bachmann, Compliance (Fn. 5), S. 93 f.; Fleischer, Corporate Compliance (Fn. 5), S. 6; ders. (Fn. 9) \$91 Rn. 61; Hölters (Fn. 12), \$ 93 Rn. 111.

187 So aber T. Fett/ S. Gebauer, Compliance-Strukturen im faktischen Bankkonzern, in: S. Grundmann et al. (Hrsg.), Unternehmensrecht zu Beginn des 21. Jahrhunderts, Festscshrift für Eberhard Schwark zum 70. Geburtstag, München 2009, S. 375 (385) (für das Aufsichtsrecht); Bürkle (Fn. 2), $\$ 8$ Rn. 65.

188 Hierzu Spindler, Vorstandspflichten (Fn. 9), Rn. 61 f.; ders., Bankengruppe (Fn. 7), S. 905 (915); Fett/ Theusinger, Compliance (Fn. 172), S. 6 (8 f.); ebenso im Ansatz U.H. Schneider/S.H. Schneider, Konzern-Compliance (Fn. 118), S. 2061 (2061 ff.); Fleischer, Corporate Compliance (Fn. 5), S. 3 f.; Kremer/Klahold, Industriekonzerne (Fn. 107), S. 113 (127); Verse, Legalitätskontrollpflichten (Fn. 5), S. 407 ff.; von Busekist/Hein, Mindestanforderungen (Fn. 8), S. 47; J. Koch, Unternehmensverbund (Fn. 8), S. 1019; Grundmeier, Konzern (Fn. 169), S. 487 (500); für die Einrichtungspflicht eines konzernweiten Kontrollsystems in der GmbH OLG Jena NZG 2010, S. 226, dazu Klindt/Pelz/Theusinger, Compliance (Fn. 139), S. 2385 (2386); eine konzernweite Compliance Pflicht bejahend auch Reichert/Ott, Vorstandspflichten (Fn. 5), S. 2173 ff.; Bicker, Umsetzung (Fn. 5), S. 548 ff.; offen lassend Schneider, Compliance (Fn. 5), S. 1325.

189 Fleischer, Corporate Compliance (Fn. 5), S. 6; ders. (Fn.9), \$91 Rn. 60; Schneider, Compliance (Fn. 5), S. 1325 f.; Grundmeier, Konzern (Fn. 169), S. 487 (500).

190 T. Kremer/ C. Klahold, Risikobereich und Haftung: Compliance in Industrieunternehmen, in: G. Krieger/U. H. Schneider (Hrsg.), Handbuch Managerhaftung, Köln 2007, \$18 Rn. 49 ff.; Fleischer, Corporate Compliance (Fn. 5), S. 6; ders. (Fn. 9), \$91 Rn. 61.

191 Bürkle (Fn. 2), $\mathbb{S} 8$ Rn. 69; Fleischer, Corporate Compliance (Fn. 5), S. 5; ders. (Fn. 9) $\$ 91$ Rn. 59.

192 S.H. Schneider, Informationspflichten und Informationssystemeinrichtungen im Aktienkonzern, Berlin 2006, S. 310 ff.; U.H. Schneider/ S.H. Schneider, Konzern-Compliance (Fn. 118), S. 2061 (2065); Fleischer, Corporate Compliance (Fn. 5), S. 6 und ders. (Fn. 9), $\$ 91$ Rn. 61, je mit Verweis auf S. Gebauer/S. Niermann, Corporate Compliance (Fn. 2), $\$ 36$ Rn. 70 (für $\$ 25 a \mathrm{KWG);} \mathrm{Schneider,}$ Compliance (Fn. 5), S. 1326; Lutter, FS Goette (Fn. 162), 2011, S. 289, 2923. 
gelmäßig, als auch anlassbezogen zu erfolgen hätte, ${ }^{193}$ hängt wiederum von den rechtlichen Einflussmöglichkeiten ab. ${ }^{194}$ Zumindest ist die Einrichtung dieses Informationssystems bei vorhandener Weisungsmöglichkeit nach $\$ 308$ Abs. 1 AktG in Regel zulässig; denn durch eine Weisung lässt sich die untergeordnete Gesellschaft zur Informationsherausgabe verpflichten, ${ }^{195}$ was ebenso sämtliche, zur Ausgestaltung einer konzernweiten Compliance-Organisation notwendigen Informationen umfassen kann. ${ }^{196}$ Demgegenüber kann im faktischen Konzern schon durch die Informationsweitergabe ein Nachteil iSd. $\$ 311$ AktG für das Tochterunternehmen entstehen, ohne dass für den erfolgten Verstoß gegen die Pflicht zu rechtmäßigem Handeln durch die Datenherausgabe ein entsprechender Ausgleich erfolgen könnte. ${ }^{197}$ Ebenso wenig kann aus $\$ 294$ Abs. 3 S. 2 HGB oder aus $\mathbb{9} 91$ Abs. 2 AktG ein allgemeiner Informationsanspruch der Muttergesellschaft abgeleitet werden, ${ }^{198}$ sodass im faktischen Konzern ein umfassendes Informationssystem an seine rechtlichen Grenzen stößt. ${ }^{199}$ Zusammengefasst haben sich die Compliance-Pflichten im Unternehmensverbund an der jeweiligen Konzernierungsform auszurichten. ${ }^{200}$

Auf der anderen Seite entlastet eine konzernweite Compliance-Organisation die Vorstände der untergeordneten Gesellschaften nicht völlig von ihrer eigenen Pflicht, sich um eine Compliance im eigenen Unternehmen zu kümmern; allerdings kann sich bei einer konzernweiten Compliance (z.B. im Vertragskonzern) die Vorstandsverantwortlichkeit in Überwachungs- und Kontrollpflichten umwandeln. ${ }^{201} \mathrm{Zu}$ den einzubeziehenden Gesellschaften gehören aber nicht nur die nationalen Tochtergesellschaften, sondern auch ausländische Untergesellschaften. ${ }^{202}$

193 Kremer/Klahold (Fn. 190), $\$ 18$ Rn. 45 ff.; Fleischer, Corporate Compliance (Fn. 5), S. 6; ders. (Fn. 9), $\mathbb{9} 91 \mathrm{Rn} .61$.

194 Gegen eine Verpflichtung auch Hölters (Fn. 12), \$93 Rn. 111.

195 H. Hirte in: K. J. Hopt/ H. Wiedemann (Hrsg.), Großkommentar zum Aktiengesetz, Bd. 8, 4. Aufl., Berlin 2013, \308 Rn. 29; Krieger (Fn. 181) \70 Rn. 151; Emmerich Fn. (180), \308 AktG Rn. 39; R. Veil, in: G. Spindler/E. Stilz (Hrsg.), Kommentar zum Aktiengesetz, Band I, 2. Aufl., München 2010, $\$ 308$ Rn. 20.

196 Lutter, FS Goette (Fn. 162), S. 289 (293); ähnlich M. Habersack, Gedanken zur konzernweiten Compliance-Verantwortung des Geschäftsleiters eines herrschenden Unternehmens, in: S. Bechtold et al. (Hrsg.), FS Möschel (Fn. 169), S. 1175 (1188ff.); Tröger, Konzernverantwortung (Fn. 185) S. 475 (503).

197 F. Bauckhage-Hoffer/ P. Katko, Compliance-Systeme und Datentransfer im Konzern, WM 2012, S. 486 (487f.).

198 Bauckhage-Hoffer/Katko, Compliance-Systeme (Fn. 197), S. 486 (487f.); zu \294 Abs. 3 HGB: B. Singhof, Zur Weitergabe von Insiderinformationen im Unterordnungskonzern, ZGR 2001, S. 146 (156 f.); Wundenberg, Compliance (Fn. 50), S. 183.

199 I.E. wohl auch Lutter, FS Goette (Fn. 162), S. 289 (294).

200 Dreher, Kartellrechtscompliance (Fn. 3), S. 102 f.; Bürkle (Fn. 2), $\$ 8$ Rn. 65; Fleischer, Corporate Compliance (Fn. 5), S. 8; ders. (Fn. 9), $\$ 91$ Rn. 62; Spindler,Bankengruppe (Fn. 7), S. 916.

201 Ähnlich Dreher, Kartellrechtscompliance (Fn. 3), S. 104; Spindler, Bankengruppe (Fn. 7), S. 916.

202 J. Bürkle, Corporate Compliance als Standard guter Unternehmensführung des Deutschen Corporate Governance Kodex, BB 2007, S. 1797 (1799); Fleischer, Corporate Compliance (Fn. 5), S. 6; Lutter, FS Goette (Fn. 162), S. 289 (294). 


\section{Datenschutzrechtliche Grenzen}

Im Rahmen der Informationsbeschaffung dürfen ferner nicht die datenschutzrechtlichen Schranken der Informationsweitergabe übersehen werden. ${ }^{203}$ Denn im Fall personenbezogener Daten ist nicht etwa der Konzern selbst Adressat der datenschutzrechtlichen Pflichten, sondern jede einzelne Konzerngesellschaft; der Konzern wird als rechtliche Vielheit betrachtet. ${ }^{204}$ Da der konzerninterne Informationsfluss somit genauso wie der Informationsfluss zwischen Dritten iSd. $\int 3$ Abs. 8 S. 2 BDSG behandelt wird, ${ }^{205}$ dürfen sich die Konzerngesellschaften intern nicht ohne Rechtfertigung untereinander bei den personenbezogenen Daten anderer Gesellschaften bedienen, ${ }^{206}$ selbst wenn es sich um eine Eingliederung iSd. $\mathbb{S} 319 \mathrm{ff}$. AktG handelt. $^{207}$ Der konzerninterne Informationsaustausch hängt damit von einem Rechtfertigungstatbestand des BDSG ab, sodass das BDSG die gesellschaftsrechtlichen Möglichkeiten der Konzernausgestaltungen beschränkt. ${ }^{208}$ Dafür kommen zunächst die Auftragsdatenverarbeitung gem. $\mathbb{1} 11$ BDSG bzw. Art. 16, 17 Abs. 2, 3 Datenschutz-RL in Betracht, ${ }^{209}$ vor allem aber die Rechtfertigungsgründe nach $\mathbb{S} \mathbb{S} 28 \mathrm{ff}$. BDSG, ${ }^{210}$ was einen einzelfallunabhängigen Datenaustausch ausschließt. ${ }^{211}$ Ein allgemeines Konzerninteresse wird hierzu in der Regel nicht ausreichen, da sonst die grundlegende Entscheidung, den Konzern nicht als Einheit zu behandeln, unterlaufen würde. ${ }^{212}$ Vielmehr ist dazu ein spezielles Interesse einer Konzerngesellschaft not-

203 Eingehend G. Spindler, Konzerninterne Informationsflüsse und Datenschutz - de lege lata und de lege ferenda, in: G. Krieger et al. (Hrsg.), FS Hoffmann-Becking (Fn. 107), S. 1185 ff.

204 Bauckhage-Hoffer/Katko, Compliance-Systeme (Fn. 197), S. 486 (488); dazu G. Spindler, Konzerninterne Informationsflüsse und Datenschutz - de lege lata und de lege ferenda, in: G. Krieger et al. (Hrsg.), FS Hoffmann-Becking (Fn. 107), S. 1185 (1186).

205 S. Simitis, in: S. Simitis (Hrsg.), Kommentar zum Bundesdatenschutzgesetz, 7. Aufl., Baden-Baden 2011, $\$ 2$ Rn. 142; P. Gola/ R. Schomerus, in: P. Gola/ R. Schomerus (Hrsg.), Kommentar zum Bundesdatenschutzgesetz, 11. Aufl., München 2012, \2 Rn. 21, \$27 Rn. 4 f.; Thüsing, Arbeitnehmerdatenschutz und Compliance, München 2010, Rn. $412 \mathrm{f}$.

206 H.-H. Schild/ M.-T. Tunefeld, Datenverarbeitung im internationalen Konzern, DuD 2011, S. 629 (631).

207 Dammann (Fn. 105), $\ 3$ Rn. 232; dazu Spindler, FS Hoffmann-Becking (Fn. 107), S. 1185 (1186).

208 Dazu Spindler, FS Hoffmann-Becking (Fn. 107), S. 1185 (1187f.).

209 Richtlinie 95/46/EG des Europäischen Parlaments und des Rates vom 24. Oktober 1995 zum Schutz natürlicher Personen bei der Verarbeitung personenbezogener Daten und zum freien Datenverlehr (EG-Datenschutzrichtlinie).

210 Ehmann (Fn. 205), $\mathbb{1} 10$ Rn. $96 \mathrm{f}$.

211 Schild/Tinnefeld, (Fn. 206) S. 629 (631); dazu G. Spindler, Konzerninterne Informationsflüsse und Datenschutz - de lege lata und de lege ferenda, in: G. Krieger et al. (Hrsg.), FS Hoffmann-Becking (Fn. 107), S. 1185 (1188 f.).

212 Dazu G. Spindler, Konzerninterne Informationsflüsse und Datenschutz - de lege lata und de lege ferenda, in: G. Krieger et al. (Hrsg.), FS Hoffmann-Becking (Fn. 107), S. 1185 (1192 f.) mwN. 
wendig, um den Anforderungen des $\mathbb{2} 28$ Abs. 2 Nr. 2 a) gerecht zu werden. ${ }^{213} \mathrm{Im}$ Ergebnis kann eine Datenübermittlung nach $\$ 28$ Abs. 2 Nr. 2 a) BDSG daher nur im Einzelfall bei Vorliegen eines berechtigten Interesses gegeben sein. ${ }^{214}$

Ebenso wenig können Verträge iSd. $\mathbb{S} 291 \mathrm{ff}$. AktG als Verträge nach $\mathbb{} 28$ Abs. 1 S. 1 Nr. 1 BDSG qualifiziert werden; ${ }^{215}$ wiederum würde sonst die Betrachtung des Konzerns als „Dritter“ umgangen. ${ }^{216}$ Datenübermittlungen und -verarbeitungen können weiterhin durch Einwilligungen nach $\mathbb{\$} 4 \mathrm{a}$ Abs. 1 BDSG und $\mathbb{S} 4 \mathrm{c}$ Abs. 1 S. 1 Nr. 1 BDSG bzw. Art. 7 a) iVm. Art. 2 h) Datenschutz-RL gerechtfertigt sein, wobei deren hohen Anforderungen nicht dazu führen können, dass eine Datennutzung im Konzern generell gerechtfertigt ist, sondern stets die vorherige Festlegung von bestimmten Informationsempfängern zu bestimmten Zwecken erfordert. ${ }^{217}$ In diesem Sinne werden auch teilweise die Betriebsvereinbarungen als „andere Rechtsvorschriften “ iSd. $\mathbb{S} 4$ Abs. 1 BDSG ausgelegt, ${ }^{218}$ und in der Rechtsprechung ${ }^{219}$ zur Rechtfertigung von Datenverarbeitungen herangezogen - was allerdings nicht ganz zweifelsfrei ist. ${ }^{220}$

Insgesamt kann eine Compliance-Organisation daher nicht ohne weiteres personenbezogene Daten erheben und übermitteln, sondern hat sich zunächst der Mittel von aggregierten oder pseudonymisierten Daten zu bedienen, bevor die Verfolgung von

213 Simitis (Fn. 205), $\$ 28$ Rn. 177 mit Verweis auf H. v. Sponeck, Weitergabe von Personaldaten innerhalb des Konzerns, CR 1991, S. 600; T. Lambrich/ N. Cahlik, Austausch von Arbeitnehmerdaten in multinationalen Konzernen - Datenschutz- und betriebsverfassungsrechtliche Rahmenbedingungen -, RDV 2002, S. 287 (291); näher G. Spindler, Konzerninterne Informationsflüsse und Datenschutz - de lege lata und de lege ferenda, in: G. Krieger et al. (Hrsg.), FS Hoffmann-Becking (Fn. 107), S. 1185 (1193) mwN.

214 Simitis (Fn. 205), $\$ 2$ Rn. 158; Ruppmann, Der konzerninterne Austausch personenbezogener Daten, Baden-Baden 2000, S. 63.

215 So aber R. Niedermeier/S. Schröcker, Die „Homogene Datenschutzzelle“, RDV 2001, S. 90 (96ff.).

216 Simitis (Fn. 205), $\mathbb{S} 2$ Rn. 150.

217 Ähnlich zur Einwilligung Bauckhage-Hoffer/Katko, Compliance-Systeme (Fn. 197), S. 486 (488 f.); G. Spindler, Konzerninterne Informationsflüsse und Datenschutz - de lege lata und de lege ferenda, in: G. Krieger et al. (Hrsg.), FS Hoffmann-Becking (Fn. 107), S. 1185 (1195 f.).

218 BAG NZA 1996, S. 945 (947); BAG NZA 1986, S. 643 (646); Simitis (Fn. 205), \$2 Rn. 160; Sokol (Fn. 205), $\mathbb{} 4$ Rn. 11; J. Taeger, in: J. Taeger/ D. Gabel (Hrsg.), Kommentar zum Bundesdatenschutzgesetz und zu den Datenschutzvorschriften des TKG und TMG, Frankfurt am Main 2010, \$ 4 Rn. 34; C. Klug, Globaler Arbeitnehmerdatenschutz - Ausstrahlungswirkung der EG-Datenschutzrichtlinie auf Drittländer am Beispiel der USA, RDV 1999, S. 109 (113); M. D. Hilber, Die datenschutzrechtliche Zulässigkeit intranet-basierter Datenbanken internationaler Konzerne, RDV 2005, S. 143 (148); Bauckhage-Hoffer/Katko, Compliance-Systeme (Fn. 197), S. 486 (489).

219 BAG NZA 1986, S. 643 (646 f.); wohl auch M. Franzen, Arbeitnehmerdatenschutz - rechtspolitische Perspektiven, RdA 2010, S. 257 (259f.); anders die Literatur Sokol (Fn. 205), \$4 Rn. 17; Golal Schomerus (Fn. 205), $\$ 4$ Rn. 10 f.; H. H. Wohlgemuth, Konfliktfälle bei grenzüberschreitender Personaldatenverarbeitung, BB 1991, S. 340 (341).

220 Näher dazu G. Spindler, Konzerninterne Informationsflüsse und Datenschutz - de lege lata und de lege ferenda, in: G. Krieger et al. (Hrsg.), FS Hoffmann-Becking (Fn. 107), S. 1185 (1198) mwNachw. 
konkreten Compliance-Verstößen eine genaue Personenbezeichnung verlangt. ${ }^{221} \mathrm{Ge-}$ rade für die präventive Erhebung von Daten iRd. Compliance-Organisation, ${ }^{222}$ insbesondere zur Aufdeckung von Straftaten Beschäftigter, kommt daher zunächst die Datenerhebung in pseudonymisierter Form in Betracht. ${ }^{223}$

\section{H. Übertragung auf andere Gesellschaftsformen.}

Wie oben dargelegt, ist die Pflicht zur Einhaltung von Rechtsnormen eine Selbstverständlichkeit - und damit auch unabhängig von der gewählten Rechtsform. Fraglich kann lediglich das Ausmaß der geforderten Compliance-Organisationspflichten sein, also das „Wie“: Anders als bei der Frage, ob auch eine GmbH eine Pflicht zur Einrichtung eines Risikomanagements trifft, was etwa bei sehr kleinen Unternehmen überzogen wäre, ${ }^{224}$ müssen auch kleine und mittlere Unternehmen Rechtspflichten einhalten, so dass hier jeweils angepasst auf die Größe des Unternehmens eine Compliance-Organisation (wenn auch ggf. nur rudimentär) anzunehmen ist. Maßgeblich ist demnach das allgemeine Entschließungs- und Auswahlermessen der Unternehmensleitung, das sich auch im GmbHG an den Vorgaben $\$ 91$ Abs. 2 AktG orientieren kann. ${ }^{225}$

\section{Nötige empirische Untersuchungen}

Die schiere Menge an geschaffenen Standards, an Publikationen und Beratungsseminaren zu dem Thema „Compliance“ suggeriert, dass es sich um eine ernsthafte Lücke in der Rechtsdurchsetzung handeln muss. Tritt man indes einen Schritt zurück und fragt sich, ob und inwieweit Compliance-Organisationen tatsächlich zu einer Reduktion der Vorfälle und Rechtsverstöße geführt haben, stellt man überrascht fest, dass es kaum ernsthafte wissenschaftliche Untersuchungen auf empirischer Basis dazu gibt. Angesichts der Flut an Publikationen und der emsigen Tätigkeit von Gesetz-

221 In Bezug auf die allg. Leitungsmacht: Simitis (Fn. 205), $\$ 28$ Rn. 177; dazu G. Spindler, Konzerninterne Informationsflüsse und Datenschutz - de lege lata und de lege ferenda, in: G. Krieger et al. (Hrsg.), FS Hoffmann-Becking (Fn. 107) S. 1185 (1193).

222 Bauckhage-Hoffer/Katko, Compliance-Systeme (Fn. 197), S. 486 (491); Zöll, in: Taeger/Gabel (Fn. 218) \$ 32 Rn. 40 ff.; Thüsing, Arbeitnehmerdatenschutz (Fn. 205), Rn. 78 ff.

223 Allgemein: Seifert (Fn. 205), \32 Rn. 118; Gola/Schomerus (Fn. 205), \32 Rn. 21; dazu G. Spindler, Konzerninterne Informationsflüsse und Datenschutz - de lege lata und de lege ferenda, in: G. Krieger et al. (Hrsg.), FS Hoffmann-Becking (Fn. 107), S. 1185 (1197 ff.).

224 P. Hommelhoff, Risikomanagement im GmbH-Recht, in: K. P. Berger et al. (Hrsg.), Festschrift für Otto Sandrock zum 70. Geburtstag, Heidelberg 2000, S. 373 (377 ff.); T. Drygalal A. Drygala, Wer braucht ein Frühwarnsystem?, ZIP 2000, S. 297 (300 f.); K. H. Maus, in: K.Schmidt/ W. Uhlenbruck (Hrsg.), Die GmbH in Krise, Sanierung und Insolvenz, 4. Aufl., Köln 2009, Rn. 1.114; R. Veil, Krisenbewältigung durch Gesellschaftsrecht, ZGR 2006, S. 374 (377f.); hierzu R. Bork, Pflichten der Geschäftsführung in Krise und Sanierung, ZIP 2011, S. 101 (105); H. Fleischer, in: H. Fleischer/ W. Goette (Hrsg.), Münchener Kommentar zum Gesetz betreffend die Gesellschaften mit beschränkter Haftung - GmbHG, Bd. II, München 2012, \$ 43 Rn. 61.

225 Drenckhan, Gläubigerschutz in der Krise der GmbH, Baden-Baden 2006, S. 60 f.; Bork, (Fn. 224), S. 101 (105); in diese Richtung auch S. Heldmann, Betrugs- und Korruptionsbekämpfung zur Herstellung von Compliance, DB 2010, S. 1235. 
gebern wie Standardsetzern wäre zu erwarten, dass den offenbar immensen Kosten einer Compliance-Organisation eine gesicherte Aussage gegenüber stünde, die den Erfolg dieser Maßnahmen belegen und die weitere Ausdifferenzierung dieser Pflichten stützen könnte. Abgesehen von seltenen auf die US-amerikanische Situation bezogenen Aussagen zur Effektivität der Compliance ${ }^{226}$ stößt man indes hier quasi in ein sozialwissenschaftlich-empirisch bzw. rechtstatsächliches „Nirvana“. Zwar führt dies noch lange nicht dazu, die Compliance-Vorschriften als verfassungsrechtlich problematisch anzusehen, da der Gesetzgeber stets einen breiten Prognosespielraum bei der Frage der Erforderlichkeit genießt; ${ }^{227}$ dennoch sollte angesichts der zunehmenden Ausweitung der organisatorischen Vorgaben die rechtstatsächliche Forschung nicht vernachlässigt werden, um nicht der Gefahr zu unterliegen, einem sich selbst verstärkenden Hype und rechtlichen „Modewelle“ hinterher zu jagen.

\section{J. Kritisches Fazit}

Abgesehen von diesem ernüchternden, da fehlenden rechtstatsächlichen Befund hinsichtlich der Kosten und Nutzen einer Compliance-Organisation sind Zweifel nicht zu verhehlen, dass zahlreiche Anforderungen letztlich nichts anderes als ,alter Wein in neuen Schläuchen" sind. Lässt man die oben skizzierten Vorgaben Revue passieren, fallen die zahlreichen Parallelen zu den schon seit Jahrzehnten in der zivilrechtlichen Rechtsprechung entwickelten Anforderungen an eine ordnungsgemäße Organisation auf, insbesondere auf der Grundlage von $\mathbb{S} \$ 823,831$ BGB, aber auch in anderen Rechtsgebieten. ${ }^{228}$ So wird stets die klare Zuordnung von Kompetenzen betont, ebenso wie Berichtswege, stichprobenartige Überwachungen etc., aber auch die Vermeidung von Interessenkollisionen bei überwachenden Stellen. ${ }^{229}$ Allerdings haben diese Pflichten durch die Verschärfung der originären Verantwortlichkeit der Organmitglieder ebenso wie die - zumindest theoretischen - Eingriffs- und Anordnungsbefugnisse vor allem im Finanzmarktbereich ein anderes Momentum erhalten. Gerade diese verwaltungsrechtlichen Möglichkeiten sind bislang indes kaum ausgelotet, auch im Hinblick auf deren konkrete Ausgestaltung. Dies gilt erst recht, wenn man eine konzernweite Ausdehnung der Compliance-Pflichten einbezieht.

$\mathrm{Ob}$ und inwieweit ein vorläufiger Höhepunkt in der weiteren Konkretisierung und Ausdehnung der Compliance-Pflichten erreicht ist, lässt sich derzeit schwer absehen. Tendenzen im Kartellrecht und auch im Strafrecht scheinen eher für eine noch größere Intensivierung zu sprechen als zuvor. Umso wichtiger erscheint es, sich erneut

226 Zur Frage, ob Compliance-Organisationen überhaupt wirksam sind J. Pape, Zur Wirksamkeit von Corporate Compliance, CCZ 2009, S. 233 unter Darstellung vier empirischer US-Studien zur Wirksamkeit und Wirksamkeitsbedingungen von Compliance Maßnahmen.

227 BVerfGE NVwZ 2011, S. 94 (102); BVerfGE 102, 197 (218); 115, 276 (309); 116, 202 (225).

228 S. dazu Spindler, Unternehmensorganisationspflichten (Fn. 17), S. 689 ff. mwNachw der älteren Judikatur; zur weiteren Entwicklung Spindler (Fn. 126) \$823 Rn. $271 \mathrm{ff}$.

229 Stellvertretend für Organisationspflichten BGH NZV 1996, S. 191 (192). 
den Grundfragen der Compliance zuzuwenden, die letztlich eine Ausprägung der allgemeinen Problematik ordnungsgemäßer Unternehmensorganisationspflichten sind, insbesondere ihrer empirischen Aufarbeitung, bevor sich die Compliance-Spirale weiter dreht. 